\title{
Analysis of nonperturbative flavor violation at chiral crossover criticality in QCD
}

\author{
Mamiya Kawaguchi $\odot,{ }^{1, *}$ Shinya Matsuzaki $\odot,{ }^{2, \dagger}$ and Akio Tomiya $\odot^{3, \$}$ \\ ${ }^{1}$ Department of Physics and Center for Field Theory and Particle Physics, Fudan University, \\ 220 Handan Road, 200433 Shanghai, China \\ ${ }^{2}$ Center for Theoretical Physics and College of Physics, Jilin University, Changchun 130012, China \\ ${ }^{3}$ RIKEN BNL Research center, Brookhaven National Laboratory, Upton, New York 11973, USA
}

(Received 28 July 2020; revised 4 January 2021; accepted 26 February 2021; published 25 March 2021)

\begin{abstract}
We discuss the violation of quark-flavor symmetry at high temperatures, induced from nonperturbative thermal loop corrections and axial anomaly, based on a three-flavor linear-sigma model including an $S U(3)$ flavor violation induced by $U(1)_{A}$ anomaly which we call an axial-anomaly induced-flavor breaking term. We employ a nonperturbative analysis following the Cornwall-Jackiw-Tomboulis formalism, and show that the model undergoes a chiral crossover with a pseudocritical temperature, consistently with lattice observations. We find following features regarding the flavor breaking eminent around and above the pseudocritical temperature: (i) up-and down-quark condensates drop faster than the strange quark's toward the criticality, but still keep nonzero value even going far above the critical temperature; (ii) the introduced anomaly-related flavor-breaking effect acts as a catalyzer toward the chiral restoration, and reduces the amount of flavor breaking in the up, down and strange quark condensates; (iii) a dramatic deformation for the meson flavor mixing structure is observed, in which the anomaly-induced favor breaking is found to be almost irrelevant; (iv) the meson spectroscopy gets corrected by the net nonperturbative flavor breaking effects, where the scalar meson mass hierarchy (inverse mass hierarchy) is significantly altered by the presence of the anomaly-related flavor breaking; (v) the topological susceptibility significantly gets the contribution from the surviving strange quark condensate, which cannot be dictated by the chiral perturbation theory, and deviates from the dilute instanton gas prediction. There the anomaly-induced flavor breaking plays a role of the destructive interference for the net flavor violation, as in the flavor breaking in the quark condensates; (vi) the $U(1)_{A}$ breaking signaled by nonzero topological susceptibility is enhanced by the nonperturbative strange quark condensate, which may account for the tension in the effective restoration of the $U(1)_{A}$ symmetry currently observed on lattices with two flavors and $2+1$ flavors near the chiral limit. Our founding critical natures can be checked in the future lattice simulations, and will give some implications to the thermal history of QCD axion.
\end{abstract}

DOI: 10.1103/PhysRevD.103.054034

\section{INTRODUCTION}

The QCD phase transition, involving the chiral symmetry restoration, is the major subject to understand the QCD vacuum structure present in the early Universe, and would also provide hints for astrophysical consequences related to the QCD thermal history, such as the QCD axion. Recent developments on lattice calculations on hot QCD,

\footnotetext{
*kawaguchi@fudan.edu.cn

†synya@jlu.edu.cn

*akio.tomiya@ riken.jp
}

Published by the American Physical Society under the terms of the Creative Commons Attribution 4.0 International license. Further distribution of this work must maintain attribution to the author(s) and the published article's title, journal citation, and DOI. Funded by SCOAP ${ }^{3}$. with $N_{f}=2+1$ flavors and physical quark masses, have revealed that the chiral phase transition is predicted as a crossover (called the chiral crossover), with the pseudocritical temperature estimated as $T_{p c} \sim 155 \mathrm{MeV}$ [1-5]. Besides, $U(1)_{A}$ symmetry is intriguing also to study the hot-QCD phase structure. The $U(1)_{A}$ symmetry breaking may crucially contribute to the chiral phase transition and its phase transition order [6]: although the $U(1)_{A}$ symmetry is broken by the quantum anomaly at low temperatures, above the chiral phase transition the fate of $U(1)_{A}$ symmetry is still mysterious, has not completely been understood yet, and left with a long-standing issue. The $U(1)_{A}$ symmetry is correlated with the nontrivialtopological vacuum structure, from which the $C P$ violation arises due to strong interactions (strong $C P$ violation) with the $\theta$ parameter. Thus the $U(1)_{A}$ anomaly at finite temperature, as well as the chiral symmetry restoration, is an 
important key to deeply understand the hot QCD vacuum including the topological structure.

The $\theta$-dependence of the QCD vacuum energy is captured in part by the topological susceptibility, which is defined as the curvature of the free energy of QCD with respect to the $\theta$ at the QCD vacuum. Note that the topological susceptibility is closely linked with the chiral symmetry breaking along with the $U(1)_{A}$ anomaly: the topological gluon configurations as the source of the axial anomaly can always be transferred by the axial rotation to current quark mass terms in the QCD generating functional, once the current quark masses are introduced in the theory. Then the topological susceptibility is given by the sum of quark condensates coupled with current quark masses and pseudoscalar susceptibilities (as will more explicitly be clarified in the later section). Thereby, the topological susceptibility at high temperatures would be a crucial quantity to explore some (effective or partial) $U(1)_{A}$ restoration in the presence of nonzero current quark masses, in relation to the hot-QCD $\theta$ vacuum structure, and the chiral symmetry restoration via quark condensates.

In the three flavor QCD at the vacuum the quark condensates are degenerated $(\langle\bar{u} u\rangle \simeq\langle\bar{d} d\rangle \simeq\langle\bar{s} s\rangle)$ as observed in the lattice simulation, $\langle\bar{s} s\rangle /\langle\bar{l} l\rangle=1.08 \pm 0.16$ $(l=u, d)$ [7]. Turning finite temperature on, this situation is dramatically altered. As has been observed in several analyses on lattice simulations for finite tempeture QCD $[8,9]$, thermal loop effects would cause a partial restoration of the chiral symmetry (or chiral crossover) at the (pseudo) critical temperature, where only the lightest $l$ quark condensate $\langle\bar{l} l\rangle$ drops more quickly than the strange quark $\langle\bar{s} s\rangle$ does. This implies a nonperturbative flavor breaking generated in hot-QCD. So, such a nonperturbative flavor breaking is expected to be reflected also in the topological susceptibility.

Actually, in Ref. [10] the authors demonstrated a significant flavor breaking effect on the topological susceptibility at high temperatures around and above the pseudocritical temperature, which was shown to be generated from nonperturbative thermal loop corrections to quark condensates, that are flavorful for light and strange quarks. There it was emphasized that the presence of strange quark contribution would be nonperturbatively crucial, which cannot be realized by the conventional chiral perturbation theory (ChPT), implying that it drives the $U(1)_{A}$ anomaly effect to be sizable even at high temperatures.

In this paper, we develop the analysis done in [10], by further investigating the flavor breaking effects on meson spectroscopy around the hot QCD criticality. We also supply the details to reach the result reported in the literature.

As was done in [10], we work on a three-flavor linearsigma model including an $S U$ (3) flavor violation induced by $U(1)_{A}$ anomaly which we call an axial-anomaly inducedflavor breaking term [11], and employ a nonperturbative analysis following the Cornwall-Jackiw-Tomboulis (CJT) formalism [12]. The nonperturbative flavor breaking is provided from two ingredients: one is nonperturbative thermal corrections, and the other is an axial anomalyinduced flavor breaking interaction.

The model is shown to undergo a chiral crossover with a pseudocritical temperature, consistently with lattice observations. Nonpertubative computation of thermal loop corrections shows that up-and down-quark condensates drop faster than the strange quark's toward the criticality, but still keep nonzero value even going far above the critical temperature, which implies the nonperturbative generation of a significant flavor breaking. It turns out that the introduced anomaly-related flavor-breaking effect acts as a catalyzer for the chiral restoration, while it reduces the amount of flavor breaking in the up, down and strange condensates.

Nonperturbative thermal corrections also generate a dramatic deformation for the meson flavor mixing structures, in passing the chiral crossover, in which the anomalyinduced favor breaking is found to be almost irrelevant. Of interest is that the meson spectroscopy gets corrected by the net nonperturbative flavor breaking effects around and above the critical temperature, where the scalar meson mass hierarchy is significantly altered by the presence of the anomaly-related flavor breaking.

We also pay particular attention to the topological susceptibility, with the correct identification keeping the flavor universal nature for the current quark masses. We find that around and above the chiral crossover, the topological susceptibility gets a nonperturbative flavor breaking via the surviving strange quark condensate, which cannot be detected by the chiral perturbation theory (ChPT), and significantly deviates from the dilute instanton gas prediction. There the anomaly-induced flavor breaking plays a role of the destructive interference for the enhancement, as in the flavor breaking in the quark condensates.

Remarkably, the $U(1)_{A}$ breaking dictated by nonzero topological susceptibility is catalyzed by the nonperturbative strange quark condensate at around the chiral crossover criticality, which may account for the tension in the effective restoration of the $U(1)_{A}$ symmetry currently observed on lattices near the chiral limit with two flavors [13-16] and $2+1$ flavors [17-19].

\section{A LINEAR SIGMA MODEL AT ZERO TEMPERATURE}

In this section, we begin by giving a brief review of the three-flavor linear-sigma model involving an axial-anomaly induced-flavor breaking proposed in the literature [11]. This model with the introduced flavor-breaking axial anomaly has been shown to successfully reproduce what is called the inverse mass hierarchy for scalar mesons lighter than $1 \mathrm{GeV}\left(m_{f_{0}(500)}<m_{\kappa(700)=K_{0}^{*}(700)}<m_{a_{0}(980)}\left(\simeq m_{f_{0}(980)}\right)\right)$. The model is built based on the chiral symmetry-which is spontaneously broken by vacuum expectation values of 
the sigma model field in a way consistent with the underlying QCD—with including the minimal flavor violation effect induced from the current quark mass difference.

\section{A. Three-flavor linear-sigma model involving the axial-anomaly induced-flavor breaking}

We start with introducing the building block, the linear sigma model field $\Phi$ as a $3 \times 3$ matrix field $\Phi$. It is parametrized by the scalar- and pseudoscalar-meson nonets as

$$
\Phi=\Phi_{a} T_{a}=\left(\sigma_{a}+i \pi_{a}\right) T_{a},
$$

where $\sigma_{a}$ are the scalar fields and $\pi_{a}$ are the pseudoscalar fields. $T_{a}=\lambda_{a} / 2(a=0,1, \ldots, 8)$ are the generators of $U(3)$, where $\lambda_{a=1, \ldots, 8}$ are the Gell-Mann matrices with $\lambda_{0}=\sqrt{2 / 3} 1_{3 \times 3}$. The generators satisfy the following identities,

$$
\begin{aligned}
\operatorname{tr}\left[T^{a} T^{b}\right] & =\frac{1}{2} \delta^{a b}, \\
{\left[T^{a}, T^{b}\right] } & =i f_{a b c} T_{c}, \\
\left\{T_{a}, T_{b}\right\} & =d_{a b c} T_{c},
\end{aligned}
$$

where $f_{a b c}$ and $d_{a b c}$ (for $a, b, c=1, \ldots, 8$ ) are the antisymmetric and symmetric structure constants of $S U(3)$ respectively, and the following conditions are satisfied

$$
f_{a b 0}=0, \quad d_{a b 0}=\sqrt{\frac{2}{3}} \delta_{a b} .
$$

Under the chiral $S U(3)_{L} \times S U(3)_{R} \times U(1)_{A}$ symmetry, the $3 \times 3$ matrix $\Phi$ transforms as

$$
\Phi \rightarrow g_{A} \cdot g_{L} \cdot \Phi \cdot g_{R}^{\dagger}
$$

where $g_{L, R} \in S U(3)_{L, R}$ and $g_{A} \in U(1)_{A}$.

The scalar mesons $\left(J^{P}=0^{+}\right)$and the pseudoscalar mesons $\left(J^{P}=0^{-}\right)$are embedded in the $\sigma_{a} T_{a}$ and $\pi_{a} T_{a}$, respectively, like

$$
\begin{gathered}
\sigma_{a} T_{a}=\frac{1}{\sqrt{2}}\left(\begin{array}{ccc}
\frac{a_{0}}{\sqrt{2}}+\frac{\sigma_{8}}{\sqrt{6}}+\frac{\sigma_{0}}{\sqrt{3}} & a^{+} & \kappa^{+} \\
a^{-} & -\frac{a_{0}}{\sqrt{2}}+\frac{\sigma_{8}}{\sqrt{6}}+\frac{\sigma_{0}}{\sqrt{3}} & \kappa^{0} \\
\kappa^{-} & \bar{\kappa}^{0} & -\frac{2 \sigma_{8}}{\sqrt{6}}+\frac{\sigma_{0}}{\sqrt{3}}
\end{array}\right), \\
\pi_{a} T_{a}=\frac{1}{\sqrt{2}}\left(\begin{array}{ccc}
\frac{\pi^{0}}{\sqrt{2}}+\frac{\eta_{8}}{\sqrt{6}}+\frac{\eta_{0}}{\sqrt{3}} & \pi^{+} & K^{+} \\
\pi^{-} & -\frac{\pi^{0}}{\sqrt{2}}+\frac{\eta_{8}}{\sqrt{6}}+\frac{\eta_{0}}{\sqrt{3}} & K^{0} \\
K^{-} & \bar{K}^{0} & -\frac{2 \eta_{8}}{\sqrt{6}}+\frac{\eta_{0}}{\sqrt{3}}
\end{array}\right),
\end{gathered}
$$

where $\pi^{ \pm}=\left(\pi_{1} \mp i \pi_{2}\right) / \sqrt{2}$ and $\pi^{0}=\pi_{3}$ are pions, $K^{ \pm}=\left(\pi_{4} \mp i \pi_{5}\right) / \sqrt{2}, \quad K^{0}=\left(\pi_{6}-i \pi_{7}\right) / \sqrt{2}$ and $\bar{K}^{0}=$ $\left(\pi_{6}+i \pi_{7}\right) / \sqrt{2}$ are kaons, $\eta_{0}=\pi_{0}$ and $\eta_{8}=\pi_{8}$ are admixtures of the $\eta$ meson and $\eta^{\prime}$. In the scalar meson nonet, the $a_{0}(980)$ meson is identified as the isotriplet component, $a^{ \pm}=\left(\sigma_{1} \mp i \sigma_{2}\right) / \sqrt{2}$ and $a_{0}=\sigma_{3}$. The $\kappa(700)=K_{0}^{*}(700)$ mesons, forming the isodoublet (including single strange quark as valence quarks), are assigned as $\kappa^{ \pm}=$ $\left(\sigma_{4} \mp i \sigma_{5}\right) / \sqrt{2}, \kappa^{0}=\left(\sigma_{6}-i \sigma_{7}\right) / \sqrt{2}$ and $\bar{\kappa}^{0}=\left(\sigma_{6}+i \sigma_{7}\right) / \sqrt{2}$. The $\sigma_{0}$ and $\sigma_{8}$ are referred to as the admixtures of the $f_{0}(500)$ and $f_{0}(980)$.

With the linear sigma model field $\Phi$ as the building block at hand, the three-flavor linear sigma model is written down

$$
\mathcal{L}=\operatorname{tr}\left[\partial_{\mu} \Phi \partial^{\mu} \Phi^{\dagger}\right]-V .
$$

where $V$ represents the potential terms,

$$
V=V_{0}+V_{\text {anom }}+V_{\text {anom }}+V_{\mathrm{SB}}+V_{\mathrm{SB}-\text { anom }} .
$$

$V_{0}$ is an invariant part under the $S U(3)_{L} \times S U(3)_{R} \times$ $U(1)_{A}$ symmetry, in which the mass term of $\Phi$ and the fourpoint interaction terms are incorporated,

$V_{0}=\mu^{2} \operatorname{tr}\left[\left(\Phi^{\dagger} \Phi\right)\right]+\lambda_{1} \operatorname{tr}\left[\left(\Phi^{\dagger} \Phi\right)^{2}\right]+\lambda_{2}\left(\operatorname{tr}\left[\left(\Phi^{\dagger} \Phi\right)\right]\right)^{2}$,

where $\mu^{2}$ can take either a positive or negative value and $\lambda_{1,2}$ are dimensionless quartic coupling constants.

The $U(1)_{A}$ anomalous part, but keeping the chiral $S U(3)_{L} \times S U(3)_{R}$ symmetry, is incorporated in $V_{\text {anom }}$. The lowest dimensional operator for the $U(1)_{A}$ anomalous term is given by a la Kobayashi-Maskawa-'t Hooft (KMT) [20-23],

$$
V_{\text {anom }}=-B\left(\operatorname{det}[\Phi]+\operatorname{det}\left[\Phi^{\dagger}\right]\right),
$$

where the parameter $B$ is real, having mass dimension one. This $V_{\text {anom }}$ is necessary to supply the sufficiently large mass for the $\eta^{\prime}$ meson to be no longer a NambuGoldstone boson.

The explicit chiral symmetry breaking originates from the current quark mass matrix $\mathcal{M}=\operatorname{diag}\left(m_{u}, m_{d}, m_{s}\right)$ in the underlying QCD Lagrangian. We incorporate this explicit breaking structure into the linear sigma model, by regarding the current quark mass matrix $\mathcal{M}$ as a spurion field, which transforms in the same way as $\Phi$ does, $\mathcal{M} \rightarrow g_{A} \cdot g_{L} \cdot \mathcal{M} \cdot g_{R}^{\dagger}$ and develops the vacuum expectation value $\langle\mathcal{M}\rangle=\operatorname{diag}\left\{m_{u}, m_{d}, m_{s}\right\}$. Thus the explicit breaking terms can be introduced in the linear-sigma model-Lagrangian in a chiral-invariant way. In the minimal flavor violation limit where only the one $\mathcal{M}$ can be operative, the $V_{\mathrm{SB}}$ part, free from the nonperturbative axial anomaly, thus goes like 


$$
V_{\mathrm{SB}}=-c \operatorname{tr}\left[\mathcal{M} \Phi^{\dagger}+\mathcal{M}^{\dagger} \Phi\right],
$$

where the parameter $c$ is taken to be real, and has mass dimension two. The parameter $c$ will not solely show up in the physical quantities, because its degree of freedom corresponds to the renormalization scale ambiguity in defining quark condensates.

The $V_{\text {SB-anom }}$ is the axial-anomaly induced-flavor breaking term, introduced in [11]. In the minimal flavor violation limit where the single $\mathcal{M}$ is only allowed to be inserted, the $V_{\text {SB-anom }}$ is cast is into the form, ${ }^{1}$

$$
V_{\mathrm{SB}-\mathrm{anom}}=-k c\left[\epsilon_{a b c} \epsilon^{d e f} \mathcal{M}_{d}^{a} \Phi_{e}^{b} \Phi_{f}^{c}+\text { H.c. }\right] \text {, }
$$

where the parameter $k$ is real, having mass dimension -1 , and $\epsilon_{a b c}$ is totally antisymmetric tensor under the exchange of indices $a, b$ and $c$ with $\epsilon_{123}=1$. It has been demonstrated [11] that, because of the flavor-singlet feature, this $k$ term supplies the contribution $\propto m_{s}$ only to the mass of $a_{0}(980)$ meson, while the $K_{0}^{*}(700)$ gets the term $\propto m_{u, d}$, so it plays the crucial role of realizing the inverse mass hierarchy for the scalar mesons lighter than $1 \mathrm{GeV}$ : $m\left[a_{0}(980)\right] \simeq m\left[f_{0}(980)\right]>m\left[K_{0}^{*}(700)\right]>m\left[f_{0}(500)\right]$.

\section{B. Spontaneous chiral symmetry breaking, meson masses, and topological susceptibility at zero temperature}

In the linear sigma model, the spontaneous chiral symmetry breaking occurs by the nonzero vacuum expectation value of the $\Phi$ field:

$$
\langle\Phi\rangle=\bar{\Phi}=T_{a} \bar{\sigma}_{a} .
$$

We impose the isospin symmetry, $m_{l} \equiv m_{u}=m_{d} \neq m_{s}$, so that the vacuum expectation values $\bar{\sigma}_{a}$ are taken as

$$
\bar{\sigma}_{a} T_{a}=\bar{\sigma}_{0} T_{0}+\bar{\sigma}_{8} T_{8}=\operatorname{diag}\left(\bar{\Phi}_{1}, \bar{\Phi}_{1}, \bar{\Phi}_{3}\right)
$$

where

$$
\begin{aligned}
& \bar{\Phi}_{1}=\frac{1}{\sqrt{6}} \bar{\sigma}_{0}+\frac{1}{2 \sqrt{3}} \bar{\sigma}_{8}, \\
& \bar{\Phi}_{3}=\frac{1}{\sqrt{6}} \bar{\sigma}_{0}-\frac{1}{\sqrt{3}} \bar{\sigma}_{8} .
\end{aligned}
$$

For the background fields $\bar{\sigma}_{0,8}$, the potential Eq. (2.7) is evaluated as

$$
\begin{aligned}
V(\bar{\sigma})= & \frac{1}{2} \mu^{2}\left(\bar{\sigma}_{0}^{2}+\bar{\sigma}_{8}^{2}\right)+\lambda_{1}\left[\frac{1}{12} \bar{\sigma}_{0}^{4}+\frac{1}{2} \bar{\sigma}_{0}^{2} \bar{\sigma}_{8}^{2}-\frac{1}{3 \sqrt{2}} \bar{\sigma}_{0} \bar{\sigma}_{8}^{3}+\frac{1}{8} \bar{\sigma}_{8}^{4}\right]+\frac{\lambda_{2}}{4}\left(\bar{\sigma}_{0}^{2}+\bar{\sigma}_{8}^{2}\right)^{2} \\
& -B\left[\frac{1}{3 \sqrt{6}} \bar{\sigma}_{0}^{3}-\frac{1}{2 \sqrt{6}} \bar{\sigma}_{0} \bar{\sigma}_{8}^{2}-\frac{1}{6 \sqrt{3}} \bar{\sigma}_{8}^{3}\right]-\sqrt{\frac{2}{3}}\left(2 c m_{l}+c m_{s}\right) \sigma_{0}-\frac{2}{\sqrt{3}}\left(c m_{l}-c m_{s}\right) \sigma_{8} \\
& -k c\left[\frac{m_{l}}{3}\left(4 \bar{\sigma}_{0}^{2}-2 \sqrt{2} \bar{\sigma}_{0} \bar{\sigma}_{8}-4 \bar{\sigma}_{8}^{2}\right)+\frac{m_{s}}{3}\left(2 \bar{\sigma}_{0}^{2}+2 \sqrt{2} \bar{\sigma}_{0} \bar{\sigma}_{8}+\bar{\sigma}_{8}^{2}\right)\right] .
\end{aligned}
$$

From this potential, the stationary conditions for $\bar{\sigma}_{0}$ and $\bar{\sigma}_{8}$ read

$$
\begin{aligned}
c \sqrt{\frac{2}{3}\left(2 m_{l}+m_{s}\right)=} & \bar{\sigma}_{0}\left[\mu^{2}+\frac{\lambda_{1}}{3}\left(\bar{\sigma}_{0}\right)^{2}+\lambda_{2}\left(\bar{\sigma}_{0}\right)^{2}-\frac{B}{\sqrt{6}} \bar{\sigma}_{0}\right]+\left(\bar{\sigma}_{8}\right)^{2}\left[\lambda_{1} \bar{\sigma}_{0}+\lambda_{2} \bar{\sigma}_{0}-\frac{\lambda_{1}}{3 \sqrt{2}} \bar{\sigma}_{8}+\frac{B}{2 \sqrt{6}}\right] \\
& -k c\left[\frac{m_{l}}{3}\left(8 \bar{\sigma}_{0}-2 \sqrt{2} \bar{\sigma}_{8}\right)+\frac{m_{s}}{3}\left(4 \bar{\sigma}_{0}+2 \sqrt{2} \bar{\sigma}_{8}\right)\right], \\
c \frac{2}{\sqrt{3}}\left(m_{l}-m_{s}\right)= & \bar{\sigma}_{8}\left[\mu^{2}+\frac{B}{\sqrt{6}} \bar{\sigma}_{0}+\frac{B}{2 \sqrt{3}} \bar{\sigma}_{8}+\left(\lambda_{1}+\lambda_{2}\right) \bar{\sigma}_{0}^{2}-\frac{\lambda_{1}}{\sqrt{2}} \bar{\sigma}_{0} \bar{\sigma}_{8}+\left(\frac{\lambda_{1}}{2}+\lambda_{2}\right)\left(\bar{\sigma}_{8}\right)^{2}\right] \\
& -k c\left[\frac{m_{l}}{3}\left(-2 \sqrt{2} \bar{\sigma}_{0}-8 \bar{\sigma}_{8}\right)+\frac{m_{s}}{3}\left(2 \sqrt{2} \bar{\sigma}_{0}+2 \bar{\sigma}_{8}\right)\right] .
\end{aligned}
$$

\footnotetext{
${ }^{1}$ A field redefinition for the linear sigma model field $\Phi$ (or the spurion field $\mathcal{M}$ ) can eliminate the $k$-term, however, instead would necessarily yield terms including higher orders in $\mathcal{M}$. Hence, the field redefinition will be off from the criterion of the minimal flavor violation, on which the present model is based. On this basis, it is robust that the $k$ term is only the source related to the anomaly-related flavor breaking. Though being not associated with the U(1) axial anomaly (which turns out to be manifest in the limit of nonlinear realization, i.e., when reduced back to the chiral perturbation theory), introduction of terms $\operatorname{like} \operatorname{tr}\left[\mathcal{M} \Phi^{\dagger} M \Phi^{\dagger}+\mathrm{H} . c.\right]$ and $\operatorname{tr}\left[\mathcal{M} \Phi^{\dagger}\right] \operatorname{tr}\left[\Phi \Phi^{\dagger}\right]+$ H.c., will not be contradicted with our criterion of the minimal flavor violation, hence might make some effects on what we have argued on the inverse mass hierarchy, in addition to the $k$-term. The extended analysis including those operators will be pursued elsewhere.
} 


\section{Scalar and pseudoscalar meson masses}

Around the vacuum given by $\bar{\sigma}_{0,8}$ in Eq. (2.16), the scalar mesons arise as the fluctuating modes, to form the mass matrix $\left(m_{S}^{2}\right)_{i j}(i, j=0,1,4,8)$, with the matrix elements,

$$
\begin{aligned}
\left(m_{S}^{2}\right)_{00}= & \mu^{2}+\lambda_{1}\left(\bar{\sigma}_{0}^{2}+\bar{\sigma}_{8}^{2}\right)+\lambda_{2}\left(3 \bar{\sigma}_{0}^{2}+\bar{\sigma}_{8}^{2}\right) \\
& -B \sqrt{\frac{2}{3}} \bar{\sigma}_{0}-\frac{4}{3} k c\left(2 m_{l}+m_{s}\right), \\
\left(m_{S}^{2}\right)_{88}= & \mu^{2}+\lambda_{1}\left[\bar{\sigma}_{0}^{2}-\sqrt{2} \bar{\sigma}_{0} \bar{\sigma}_{8}+\frac{3}{2} \bar{\sigma}_{8}^{2}\right]+\lambda_{2}\left(\bar{\sigma}_{0}^{2}+3 \bar{\sigma}_{8}^{2}\right) \\
& +\frac{B}{\sqrt{3}}\left(\frac{1}{\sqrt{2}} \bar{\sigma}_{0}+\bar{\sigma}_{8}\right)+\frac{2}{3} k c\left(4 m_{l}-m_{s}\right), \\
\left(m_{S}^{2}\right)_{08}= & \lambda_{1}\left[2 \bar{\sigma}_{0} \bar{\sigma}_{8}-\frac{1}{\sqrt{2}} \bar{\sigma}_{8}^{2}\right]+2 \lambda_{2} \bar{\sigma}_{0} \bar{\sigma}_{8}+\frac{B}{\sqrt{6}} \bar{\sigma}_{8} \\
& +\frac{2 \sqrt{2}}{3} k c\left(m_{l}-m_{s}\right), \\
\left(m_{S}^{2}\right)_{11}= & \mu^{2}+\lambda_{1}\left[\bar{\sigma}_{0}^{2}+\sqrt{2} \bar{\sigma}_{0} \bar{\sigma}_{8}+\frac{1}{2} \bar{\sigma}_{8}^{2}\right]+\lambda_{2}\left(\bar{\sigma}_{0}^{2}+\bar{\sigma}_{8}^{2}\right) \\
& +\frac{B}{\sqrt{3}}\left(\frac{1}{\sqrt{2}} \bar{\sigma}_{0}-\bar{\sigma}_{8}\right)+2 k c m_{s}, \\
\left(m_{S}^{2}\right)_{44}= & \mu^{2}+\lambda_{1}\left[\bar{\sigma}_{0}^{2}-\frac{1}{\sqrt{2}} \bar{\sigma}_{0} \bar{\sigma}_{8}+\frac{1}{2} \bar{\sigma}_{8}^{2}\right]+\lambda_{2}\left(\bar{\sigma}_{0}^{2}+\bar{\sigma}_{8}^{2}\right) \\
& +\frac{B}{\sqrt{3}}\left(\frac{1}{\sqrt{2}} \bar{\sigma}_{0}+\frac{1}{2} \bar{\sigma}_{8}\right)+2 k c m_{l},
\end{aligned}
$$

where $\left(m_{S}^{2}\right)_{11}$ and $\left(m_{S}^{2}\right)_{44}$ are identified as the $a_{0}(980)$ meson and the $K_{0}^{*}(700)$ respectively, $m_{a_{0}}^{2} \equiv\left(m_{S}^{2}\right)_{11}$, $m_{\kappa}^{2} \equiv\left(m_{S}^{2}\right)_{44}$. As shown in Eq. (2.17), the $\left(m_{S}^{2}\right)_{11}$ has the $2 \mathrm{kcm}_{s}$ term, which comes from the $V_{\mathrm{SB}-\text { anom }}$ term. On the other hand, the contribution to $\left(m_{S}^{2}\right)_{44}$ from the $V_{\mathrm{SB}-\text { anom }}$ term is given as the $2 \mathrm{kcm}$ lerm. Due to $m_{s}>m_{l}$ the axialanomaly induced-flavor breaking $k$-term supplies the sufficient contribution to the $a_{0}(980)$ meson, so that the $a_{0}$ meson is necessarily heavier than the $K_{0}^{*}(700)$ meson, hence the desired inverse mass hierarchy $m_{a_{0}}>m_{\kappa}$ is realized [11].

The mixing part for $\left(m_{S}^{2}\right)_{00}$ and $\left(m_{S}^{2}\right)_{88}$ can be diagonalized by an orthogonal transformation in the following way:

$$
\begin{aligned}
\tilde{\sigma}_{i} & =\left(O_{S}^{-1}\right)_{i a} \sigma_{a}, \\
{\left[\tilde{m}_{S}^{2}\right]_{(i)} \delta_{i j} } & =\left(O_{S}^{-1}\right)_{i a}\left[m_{S}^{2}\right]_{a b}\left(O_{S}\right)_{b j},
\end{aligned}
$$

where $\left[\tilde{m}_{S}^{2}\right]_{(i)}$ are the mass eigenvalues. Thus the $f_{0}(500)$ and $f_{0}(980)$ masses are assigned and evaluated as

$$
\begin{aligned}
m^{2}\left[f_{0}(500)\right]= & \left(\tilde{m}_{S}^{2}\right)_{(0)} \\
= & \left(m_{S}^{2}\right)_{00} \cos ^{2} \theta_{S}^{0}+\left(m_{S}^{2}\right)_{88} \sin ^{2} \theta_{S}^{0} \\
& -2\left(m_{S}^{2}\right)_{08} \cos \theta_{S}^{0} \sin \theta_{S}^{0}, \\
m^{2}\left[f_{0}(980)\right]= & \left(\tilde{m}_{S}^{2}\right)_{(8)} \\
= & \left(m_{S}^{2}\right)_{00} \sin ^{2} \theta_{S}^{0}+\left(m_{S}^{2}\right)_{88} \cos ^{2} \theta_{S}^{0} \\
& +2\left(m_{S}^{2}\right)_{08} \cos \theta_{S}^{0} \sin \theta_{S}^{0},
\end{aligned}
$$

where $\theta_{S}^{0}$ represents the scalar mixing angle

$$
\theta_{S}^{0}=\frac{1}{2} \arctan \left[\frac{2\left(m_{S}^{2}\right)_{08}}{\left(m_{S}^{2}\right)_{00}-\left(m_{S}^{2}\right)_{88}}\right] .
$$

In a way similar to the scalar meson sector, the pseudoscalar mesons are also in part mixed to form the mass matrix $\left(m_{P}^{2}\right)_{i j}(i, j,=0,1,4,8)$ with the matrix elements,

$$
\begin{aligned}
\left(m_{P}^{2}\right)_{00}= & \mu^{2}+\frac{\lambda_{1}}{3}\left(\bar{\sigma}_{0}^{2}+\bar{\sigma}_{8}^{2}\right)+\lambda_{2}\left(\bar{\sigma}_{0}^{2}+\bar{\sigma}_{8}^{2}\right)+B \sqrt{\frac{2}{3}} \bar{\sigma}_{0} \\
& +\frac{4}{3} k c\left(2 m_{l}+m_{s}\right), \\
\left(m_{P}^{2}\right)_{11}= & \mu^{2}+\lambda_{1}\left[\frac{1}{3} \bar{\sigma}_{0}^{2}+\frac{\sqrt{2}}{3} \bar{\sigma}_{0} \bar{\sigma}_{8}+\frac{1}{6} \bar{\sigma}_{8}^{2}\right]+\lambda_{2}\left(\bar{\sigma}_{0}^{2}+\bar{\sigma}_{8}^{2}\right) \\
& -\frac{B}{\sqrt{3}}\left(\frac{1}{\sqrt{2}} \bar{\sigma}_{0}-\bar{\sigma}_{8}\right)-2 k c m_{s}, \\
\left(m_{P}^{2}\right)_{44}= & \mu^{2}+\lambda_{1}\left[\frac{1}{3}\left(\bar{\sigma}_{0}\right)^{2}-\frac{1}{3 \sqrt{2}} \bar{\sigma}_{0} \bar{\sigma}_{8}+\frac{7}{6}\left(\bar{\sigma}_{8}\right)^{2}\right] \\
& +\lambda_{2}\left(\bar{\sigma}_{0}^{2}+\bar{\sigma}_{8}^{2}\right)-\frac{B}{\sqrt{3}}\left(\frac{1}{\sqrt{2}} \bar{\sigma}_{0}+\frac{1}{2} \bar{\sigma}_{8}\right)-2 k c m_{l}, \\
\left(m_{P}^{2}\right)_{88}= & \mu^{2}+\lambda_{1}\left[\frac{1}{3}\left(\bar{\sigma}_{0}\right)^{2}-\frac{\sqrt{2}}{3} \bar{\sigma}_{0} \bar{\sigma}_{8}+\frac{1}{2}\left(\bar{\sigma}_{8}\right)^{2}\right] \\
& +\lambda_{2}\left(\bar{\sigma}_{0}^{2}+\bar{\sigma}_{8}^{2}\right)-\frac{B}{\sqrt{3}}\left(\frac{1}{\sqrt{2}} \bar{\sigma}_{0}+\bar{\sigma}_{8}\right) \\
& -\frac{2}{3} k c\left(4 m_{l}-m_{s}\right), \\
& \frac{2}{3} \lambda_{1} \bar{\sigma}_{0} \bar{\sigma}_{8}-\frac{\lambda_{1}}{3 \sqrt{2}} \bar{\sigma}_{8}^{2}-\frac{B}{\sqrt{6}} \bar{\sigma}_{8} \\
& -\frac{2 \sqrt{2}}{3} k c\left(m_{l}-m_{s}\right), \\
\left(m_{P}^{2}\right)_{08} & (21) \\
&
\end{aligned}
$$

where $\left(m_{P}^{2}\right)_{11}$ and $\left(m_{P}^{2}\right)_{44}$ are identified as the pion mass and the kaon mass, respectively, $m_{\pi}^{2} \equiv\left(m_{P}^{2}\right)_{11}$, $m_{K}^{2} \equiv\left(m_{P}^{2}\right)_{44}$. As the consequence of the chiral partner structure in the linear sigma model, The $\pi_{0}$ and $\pi_{8}$ mix in a similar way to the scalar meson's. The mass matrix can be diagonalized by the orthogonal transformation, 


$$
\begin{aligned}
\tilde{\pi}_{i} & =\left(O_{P}^{-1}\right)_{i a} \pi_{a}, \\
{\left[\tilde{m}_{P}^{2}\right]_{(i)} \delta_{i j} } & =\left(O_{P}^{-1}\right)_{i a}\left[m_{S, P}^{2}\right]_{a b}\left(O_{P}\right)_{b j},
\end{aligned}
$$

where the $\left[\tilde{m}_{P}^{2}\right]_{(i)}$ are the mass eigenvalues. Thus, the $\eta^{\prime}$ and $\eta$ meson masses are assigned and evaluated as

$$
\begin{aligned}
m_{\eta^{\prime}}^{2}= & \left(\tilde{m}_{P}^{2}\right)_{(0)} \\
= & \left(m_{P}^{2}\right)_{00} \cos ^{2} \theta_{P}^{0}+\left(m_{P}^{2}\right)_{88} \sin ^{2} \theta_{P}^{0} \\
& -2\left(m_{P}^{2}\right)_{08} \cos \theta_{P}^{0} \sin \theta_{P}^{0}, \\
m_{\eta}^{2}= & \left(\tilde{m}_{P}^{2}\right)_{(8)} \\
= & \left(m_{P}^{2}\right)_{00} \cos ^{2} \theta_{P}^{0}+\left(m_{P}^{2}\right)_{88} \sin ^{2} \theta_{P}^{0} \\
& +2\left(m_{P}^{2}\right)_{08} \cos \theta_{P}^{0} \sin \theta_{P}^{0},
\end{aligned}
$$

where $\theta_{P}^{0}$ denotes the pseudoscalar mixing angle at the zero temperature, which is given by

$$
\theta_{P}^{0}=\frac{1}{2} \arctan \left[\frac{2\left(m_{P}^{2}\right)_{08}}{\left(m_{P}^{2}\right)_{00}-\left(m_{P}^{2}\right)_{88}}\right] .
$$

By using the stationary condition in Eq. (2.16), the pion mass and the kaon mass can be written as

$$
\begin{aligned}
& m_{\pi}^{2}=\frac{c m_{l}}{\bar{\Phi}_{1}}\left(1+2 k \bar{\Phi}_{3}\right), \\
& m_{K}^{2}=\frac{c m_{l}+c m_{s}}{\bar{\Phi}_{1}+\bar{\Phi}_{3}}\left(1+2 k \bar{\Phi}_{1}\right) .
\end{aligned}
$$

It looks like that those masses significantly get corrections from the $k$ term having an intrinsic-flavor violation-nature associated with the determinant form (breaking the intrinsic parity). So, one might doubt if the present model surely satisfies the low-energy theorem for three-flavor case, such as the Gell-Mann-Oakes-Renner relations. To check this point, we shall look at the quark condensates, and the pion decay constant $f_{\pi}$ and the kaon decay constant $f_{K}$. The former reads

$$
\begin{gathered}
\langle\bar{l} l\rangle=\frac{\partial V(\bar{\sigma})}{\partial m_{l}}=-2 c\left(\bar{\Phi}_{1}+2 k \bar{\Phi}_{1} \bar{\Phi}_{3}\right), \\
\langle\bar{s} s\rangle=\frac{\partial V(\bar{\sigma})}{\partial m_{s}}=-2 c\left(\bar{\Phi}_{3}+2 k \bar{\Phi}_{1}^{2}\right),
\end{gathered}
$$

while the latter are read off from the overlap amplitudes between the corresponding axial-vector currents and pseudoscalars, to be given as

$$
\begin{aligned}
& f_{\pi}=2 \bar{\Phi}_{1}, \\
& f_{K}=\bar{\Phi}_{1}+\bar{\Phi}_{3} .
\end{aligned}
$$

From these, we find

$$
\begin{aligned}
f_{\pi}^{2} m_{\pi}^{2} & =-2 m_{l}\langle\bar{l} l\rangle, \\
f_{K}^{2} m_{K}^{2} & =-\frac{m_{l}+m_{s}}{2}(\langle\bar{l} l\rangle+\langle\bar{s} s\rangle),
\end{aligned}
$$

which are precisely the Gell-Mann-Oakes-Renner relations. Note an accidental cancellation of the $k$ dependence arising from both quark condensates and pion, kaon masses. Thus, the low energy theorem is intact even in the presence of the intrinsically flavor-violating $k$-term [11].

\section{Topological susceptibility: Flavor-universal nature}

We next turn to topological susceptibility $\chi_{\text {top }}$. In hot $\mathrm{QCD}$, the $\chi_{\text {top }}$ is a crucial quantity to measure the topological charge fluctuation of the QCD $\theta$-vacuum. It is defined as the curvature of the free energy of QCD with respect to the $\theta$ at the QCD vacuum with $\theta=0$ :

$$
\chi_{\text {top }}=-\left.\int_{T} d^{4} x \frac{\delta^{2} V(\theta)}{\delta \theta(x) \delta \theta(0)}\right|_{\theta=0} .
$$

where $V(\theta)$ is the $\theta$-dependent vacuum energy and the temperature integral is denoted as $\int_{T} d^{4} x \equiv \int_{0}^{1 / T} d \tau d^{3} \vec{x}$. Furthermore, the QCD $\theta$-vacuum is correlated with the presence of the $U(1)_{A}$ anomaly including quark mass contributions. As will be seen later, the $\chi_{\text {top }}$ must have a flavor-universal nature, and vanish when either of all current quark masses goes to zero, because the nonperturbative $U(1)_{A}$ anomaly induced by topological gluon configurations can always be transferred to current quark mass terms in the QCD generating functional, once the current quark masses are introduced in the theory. Thereby, the $\chi_{\text {top }}$ also plays an important role of monitoring some (effective or partial) $U(1)_{A}$ restoration in the presence of nonzero current quark masses. (That might or might not happen at high temperatures, as will be seen later.) In this section, we pay our attention to this topological susceptibility in the present linear sigma model with the anomaly-induced flavor breaking, the $k$-term.

Generically, the $\theta$-parameter can be introduced through matching the QCD generating functional with the one corresponding to the low-energy effective model which one works on. So, we shall start from the Euclidean generating functional of QCD given as a function of $\theta$,

$$
\begin{aligned}
Z_{\mathrm{QCD}}= & \int\left[\Pi_{f} d q_{f} d \bar{q}_{f}\right][d A] \exp \left[-\int_{T} d^{4} x\left\{\sum_{f}\left(\bar{q}_{L}^{f} i \gamma^{\mu} D_{\mu} q_{L}^{f}+\bar{q}_{R}^{f} i \gamma^{\mu} D_{\mu} q_{R}^{f}+\bar{q}_{L}^{f} m_{f} q_{R}^{f}+\bar{q}_{R}^{f} m_{f} q_{L}^{f}\right)\right.\right. \\
& \left.\left.+\frac{1}{4 g^{2}}\left(F_{\mu \nu}^{a}\right)^{2}+\frac{i \theta}{32 \pi^{2}} F_{\mu \nu}^{a} \tilde{F}_{\mu \nu}^{a}\right\}\right],
\end{aligned}
$$


where $q_{L(R)}^{f}$ denote the left- (right-) handed quark fields; the covariant derivative of the quark field is represented as $D_{\mu}$ involving the gluon fields $A ; F_{\mu \nu}^{a}$ is the field strength of the gluon fields with $g$ being the QCD coupling constant. The finiteness of the $\theta$-parameter indicates that the QCD system is put in the $C P$ violation domain.

Under the $U_{A}(1)$ rotation with the rotation angle $\theta_{f}$, the left- and right-handed quark fields are transformed as

$$
\begin{aligned}
q_{L}^{f} & \rightarrow \exp \left(-i \theta_{f} / 2\right) q_{L}^{f}, \\
q_{R}^{f} & \rightarrow \exp \left(i \theta_{f} / 2\right) q_{R}^{f},
\end{aligned}
$$

By rotating the chiral quark fields as above, one finds that the extra phase factor shows up in the QCD generating functional:

$$
\begin{aligned}
\int & {\left[\Pi_{f} d q_{f} d \bar{q}_{f}\right][d A] \exp \left[-\int_{T} d^{4} x\left\{\sum_{f}\left(\bar{q}_{L}^{f} i \gamma^{\mu} D_{\mu} q_{L}^{f}+\bar{q}_{R}^{f} i \gamma^{\mu} D_{\mu} q_{R}^{f}+\bar{q}_{L}^{f} m_{f} e^{i \theta_{f}} q_{R}^{f}+\bar{q}_{R}^{f} m_{f} e^{-i \theta_{f}} q_{L}^{f}\right)\right.\right.} \\
+ & \left.\left.\frac{1}{4 g^{2}}\left(F_{\mu \nu}^{a}\right)^{2}+\frac{i(\theta-\bar{\theta})}{32 \pi^{2}} F_{\mu \nu}^{a} \tilde{F}_{\mu \nu}^{a}\right\}\right],
\end{aligned}
$$

where $\bar{\theta}=\sum_{f=u, d, s} \theta_{f}=\theta_{u}+\theta_{d}+\theta_{s}$. Thus, in the presence of nonzero current quark masses, generically the $\theta$-term $\left(\theta F_{\mu \nu}^{a} \tilde{F}_{\mu \nu}^{a}\right)$ can be rotated away from the QCD generating functional by choosing the chiral rotation angle like,

$$
\theta=\bar{\theta}=\theta_{u}+\theta_{d}+\theta_{s}
$$

Instead, the $\theta$-dependence is fully absorbed into the quark mass matrix. Crucial is then to note that if either of quarks are massless, the $\theta$-dependence can be completely rotated away from the QCD generating functional. It implies that for small enough $\theta_{f}$, the $\theta_{f}$ are constrained by the flavor singlet condition [24],

$$
m_{u} \theta_{u}=m_{d} \theta_{d}=m_{s} \theta_{s} \equiv x .
$$

By using this flavor singlet condition with the choice of the parameter $\bar{\theta}$ in Eq. (2.33), the parameter $\theta_{f} \mathrm{~s}$ are determined as

$$
\theta_{u}=\frac{\bar{m}}{m_{u}} \theta, \quad \theta_{d}=\frac{\bar{m}}{m_{d}} \theta, \quad \theta_{s}=\frac{\bar{m}}{m_{s}} \theta
$$

where

$$
\bar{m}=\left(\frac{1}{m_{u}}+\frac{1}{m_{d}}+\frac{1}{m_{s}}\right)^{-1} .
$$

Thus the $\theta$-dependent vacuum energy of QCD is cast into the form

$V_{\mathrm{QCD}}(\theta)=-\ln \left[\int\left[\Pi_{f} d q_{f} d \bar{q}_{f}\right][d A] \exp \left(-\int_{T} d^{4} x \mathcal{L}_{\mathrm{QCD}}^{(\theta)}\right)\right]$

where

$$
\begin{aligned}
\mathcal{L}_{\mathrm{QCD}}^{(\theta)}= & \sum_{f}\left(\bar{q}_{L}^{f} i \gamma^{\mu} D_{\mu} q_{L}^{f}+\bar{q}_{R}^{f} i \gamma^{\mu} D_{\mu} q_{R}^{f}\right)+\bar{q}_{L} \mathcal{M}_{\theta} q_{R} \\
& +\bar{q}_{R} \mathcal{M}_{\theta}^{\dagger} q_{L}+\frac{1}{4 g^{2}}\left(F_{\mu \nu}^{a}\right)^{2}
\end{aligned}
$$

with $\mathcal{M}_{\theta}$ being the $\theta$-dependent quark matrix,

$$
\begin{aligned}
\mathcal{M}_{\theta}= & \operatorname{diag}\left[m_{u} \exp \left(i \frac{\bar{m}}{m_{u}} \theta\right)\right. \\
& \left.m_{d} \exp \left(i \frac{\bar{m}}{m_{d}} \theta\right), m_{s} \exp \left(i \frac{\bar{m}}{m_{s}} \theta\right)\right] .
\end{aligned}
$$

Therefore, from Eq. (2.29) the topological susceptibility of QCD is evaluated as

$$
\begin{aligned}
\chi_{\mathrm{top}}^{(\mathrm{QCD})}= & -\left.\int_{T} d^{4} x \frac{\delta^{2} V_{\mathrm{QCD}}}{\delta \theta(x) \delta \theta(0)}\right|_{\theta=0} \\
= & \left(\frac{\langle\bar{u} u\rangle(T)}{m_{u}}+\frac{\langle\bar{d} d\rangle(T)}{m_{d}}+\frac{\langle\bar{s} s\rangle(T)}{m_{s}}\right) \bar{m}^{2} \\
& +\int_{T} d^{4} x\left\langle\left(\sum_{f} i \bar{q}^{f}(x) \gamma_{5} q^{f}(x)\right)\right. \\
& \left.\times\left(\sum_{f} i \bar{q}^{f}(0) \gamma_{5} q^{f}(0)\right)\right\rangle \bar{m}^{2} .
\end{aligned}
$$

This is an intriguing formula having the nonperturbative correlation in QCD between the axial anomaly along with the $\theta$-vacuum and the chiral symmetry breaking. ${ }^{2}$ Note that

\footnotetext{
${ }^{2}$ The relation only between the topological susceptibility and the quark condensates was already derived in Appendix of the literature [25] by using the Ginzsparg-Wilson Dirac operator. Eq. (2.40) has extended the existing formula to finite temperature, and by compensating the pseudoscalar susceptibility part (the last two terms).
} 
the topological susceptibility goes away, if either of quarks get massless. Thus the flavor-singlet nature of the axial anomaly in QCD is surely reflected in the topological susceptibility in Eq. (2.40) [24] (see also, e.g., [26]).

As clearly shown in Appendix A, by using a couple of Ward identities, Eq. (2.40) is reduced to the well-known formula relating the $\chi_{\text {top }}$ to the disconnected part of the psuedoscalar susceptibility $\chi_{5 \text {,disc }}$ including the flavorsinglet condition. In the reduced formula, the $\chi_{5 \text {, disc }}$ implicitly includes the strange quark contribution in part, through the light-strange quark pseudoscalar susceptibility in such a way that $\chi_{5 \text {,disc }}=m_{s} /\left(2 m_{l}\right) \chi_{P}^{l s}$ [See Eq. (A11)]. In Eq. (2.40) such a strange quark contribution has been made explicit.

Even in the present linear sigma model the $\theta$-parameter must be entered only via the quark mass matrix $\mathcal{M}$, so that only the $V_{\mathrm{SB}}$ and the $V_{\mathrm{SB} \text {-anom }}$ parts include the $\theta$ dependence through $\mathcal{M}_{\theta}$ in Eq. (2.39). In the present linear sigma model, thus, the $\theta$-dependent vacuum energy $V(\theta)$ is written as

$$
\begin{aligned}
V(\theta)= & \int_{T} d^{4} x V_{\mathrm{SB}}(\theta)+\int_{T} d^{4} x V_{\mathrm{SB}-\mathrm{anom}}(\theta) \\
= & \int_{T} d^{4} x\left(-4 c m_{l} \bar{\Phi}_{1} \cos \theta_{l}-2 c m_{s} \bar{\Phi}_{3} \cos \theta_{s}\right. \\
& \left.-8 k c m_{l} \bar{\Phi}_{1} \bar{\Phi}_{3} \cos \theta_{l}-4 k c m_{s}\left(\bar{\Phi}_{1}\right)^{2} \cos \theta_{s}\right) .
\end{aligned}
$$

From Eq. (2.29), the topological susceptibility of the linear sigma model reads

$$
\begin{aligned}
\chi_{\text {top }} & =-\left.\int_{T} d^{4} x \frac{\delta^{2} V(\theta)}{\delta \theta(x) \delta \theta(0)}\right|_{\theta=0} \\
& =\left(\frac{2\langle\bar{l} l\rangle(T)}{m_{l}}+\frac{\langle\bar{s} s\rangle(T)}{m_{s}}\right) \bar{m}^{2},
\end{aligned}
$$

where the light-quark condensate $\langle\bar{l} l\rangle$ and the strange-quark condensate $\langle\bar{s} s\rangle$ should be evaluated from the linear sigma model analysis. This topological susceptibility is precisely in accordance with Eq. (2.40) at the leading order of $m_{q}$. By taking the flavor universal limit $\langle\bar{l} l\rangle=\langle\bar{s} s\rangle \equiv \Sigma$, the expression of the topological susceptibility in Eq. (2.42) can be reduced to the Leutwyler-Smiluga (LS) relation [27]: $\left.\chi_{\text {top }}\right|_{\text {LS }}=\Sigma \bar{m}$, which is derived at the leading order in the ChPT. It is remarkable that the $\chi_{\text {top }}$ includes contributions from the anomaly-induced flavor breaking $k$-term, through the quark condensates in Eq. (2.26).

\section{FORMULATION AT FINITE TEMPERATURE BASED ON THE CJT FORMALISM}

When employing perturbative evaluation of thermal loop corrections in the linear sigma model at finite temperatures, we encounter the infrared divergence, because the meson loop corrections will be drastically enhanced to overwhelm the leading order terms due to the potentially small masses of pseudo-Nambu-Goldstone bosons. Therefore, some resummation scheme is required to avoid the infrared divergence. Furthermore, performing the perturbative thermal loop calculations, some unphysical tachyonic mode would show up during a chiral phase transition. Thus, one may fail to implement the perturbative loop calculation in passing the phase boundary, which urges one to work on some nonperturbative analysis, to get reliable and physical results in the linear sigma model at finite temperatures.

In the present analysis, we shall employ the CJT formalism [12], which is well-known and a powerful nonperturbative calculation tool to study the chiral phase transition based on the linear sigma model [28,29]. The effective potential based on the CJT formalism is given as

$$
\begin{aligned}
V_{\mathrm{eff}}[\alpha, S, P]= & V(\alpha)+\frac{1}{2} \int_{k}\left\{\left[\ln S^{-1}(k)\right]_{a a}+\left[\ln P^{-1}(k)\right]_{a a}\right\} \\
& +\frac{1}{2} \int_{k}\left[\bar{S}_{a b}^{-1}(k ; \alpha) S_{b a}(k)+\bar{P}_{a b}^{-1}(k ; \alpha) P_{b a}(k)\right. \\
& \left.-2 \delta_{a b} \delta_{b a}\right]+V_{2}[\alpha, S, P],
\end{aligned}
$$

where $\alpha$ denotes a set of the expectation values of the scalar fields; $V(\alpha)$ is the tree-level potential given in Eq. (2.15); $V_{2}[\alpha, S, P]$ includes contributions from the sum of all twoparticle irreducible diagrams, in which all meson loop lines are drawn by the full (dressed) propagators, denoted as $S$ (for scalar mesons) and $P$ (pseudoscalar mesons). Here, we have used the shorthand notation for the integration based on the imaginary time formalism,

$$
\int_{k} f(k)=T \sum_{n=-\infty}^{\infty} \int \frac{d^{3} \boldsymbol{k}}{(2 \pi)^{3}} f(2 \pi i n T, \boldsymbol{k})
$$

The $\bar{S}_{a b}^{-1}(k ; \alpha)$ and $\bar{P}_{a b}^{-1}(k ; \alpha)$ are the tree-level propagators for scalar and pseudoscalar mesons respectively,

$$
\begin{aligned}
& \bar{S}_{a b}^{-1}(k ; \alpha)=-k^{2} \delta_{a b}+\left[m_{S}^{2}(\alpha)\right]_{a b}, \\
& \bar{P}_{a b}^{-1}(k ; \alpha)=-k^{2} \delta_{a b}+\left[m_{P}^{2}(\alpha)\right]_{a b},
\end{aligned}
$$

where $\left[m_{S}^{2}(\alpha)\right]_{a b}$ and $\left[m_{S}^{2}(\alpha)\right]_{a b}$ are the mass matrices of the scalar and pseudoscalar meson masses at the tree-level given in Eqs. (2.17) and (2.21). The expectation value of the one-point function $\bar{\sigma}$ and of the two-point functions denoted as $\overline{\mathcal{S}}$ and $\overline{\mathcal{P}}$ are determined from the stationary conditions, 


$$
\begin{aligned}
& \left.\frac{\delta V_{\text {eff }}[\alpha, S, P]}{\delta \alpha_{a}}\right|_{\alpha=\bar{\sigma}, S=\overline{\mathcal{S}}, P=\overline{\mathcal{P}}}=0, \\
& \left.\frac{\delta V_{\text {eff }}[\alpha, S, P]}{\delta S_{a b}}\right|_{\alpha=\bar{\sigma}, S=\overline{\mathcal{S}}, P=\overline{\mathcal{P}}}=0, \\
& \left.\frac{\delta V_{\text {eff }}[\alpha, S, P]}{\delta P_{a b}}\right|_{\alpha=\bar{\sigma}, S=\overline{\mathcal{S}}, P=\overline{\mathcal{P}}}=0 .
\end{aligned}
$$

From the latter two equations, the two-point functions can be expressed as

$$
\begin{aligned}
& \overline{\mathcal{S}}_{a b}^{-1}(k)=\bar{S}_{a b}^{-1}(k ; \bar{\sigma})+\Sigma_{a b}(k), \\
& \overline{\mathcal{P}}_{a b}^{-1}(k)=\bar{P}_{a b}^{-1}(k ; \bar{\sigma})+\Pi_{a b}(k),
\end{aligned}
$$

where $\Sigma_{a b}(k)$ and $\Pi_{a b}(k)$ are the scalar- and pseudoscalar self-energy functions,

$$
\begin{aligned}
& \Sigma_{a b}(k)=\left.2 \frac{\delta V_{2}[\alpha, S, P]}{\delta S_{b a}}\right|_{\alpha=\bar{\sigma}, S=\overline{\mathcal{S}}, P=\overline{\mathcal{P}}} \\
& \Pi_{a b}(k)=\left.2 \frac{\delta V_{2}[\alpha, S, P]}{\delta P_{b a}}\right|_{\alpha=\bar{\sigma}, S=\overline{\mathcal{S}}, P=\overline{\mathcal{P}}} \cdot
\end{aligned}
$$

$\Sigma_{a b}$ and $\Pi_{a b}$ are functions of the $\overline{\mathcal{S}}_{a b}$ and $\overline{\mathcal{P}}_{a b}$, so that Eq. (3.5) represents the Schwinger-Dyson equations for the dressed propagators of the scalar and pseudoscalar mesons.

The $V_{2}[\alpha, S, P]$ generically includes infinite number of diagrams, so an exact calculation is not practical. In the present study, we shall just pick up double-bubble diagrams, which is equivalent to the Hartree approximation. In this case, the effective potential for two-particle irreducible (2PI) diagrams $V_{2}$ is generated from the four-point interactions of the $\lambda_{1,2}$ terms in Eq. (2.8), and takes the form

$$
V_{2}[S, P]=F_{a b c d}\left[\int_{k} S_{a b}(k) \int_{p} S_{c d}(p)+\int_{k} P_{a b}(k) \int_{p} P_{c d}(p)\right]+2 H_{a b c d} \int_{k} S_{a b}(k) \int_{p} P_{c d}(p) .
$$

where

$$
\begin{aligned}
& F_{a b c d}=\frac{\lambda_{1}}{8}\left(d_{a b n} d_{n c d}+d_{a d n} d_{n b c}+d_{a c n} d_{n b d}\right)+\frac{\lambda_{2}}{4}\left(\delta_{a b} \delta_{c d}+\delta_{a d} \delta_{b c}+\delta_{a c} \delta_{b d}\right) \\
& H_{a b c d}=\frac{\lambda_{1}}{8}\left(d_{a b n} d_{n c d}+f_{a c n} f_{n b d}+f_{b c n} f_{n a d}\right)+\frac{\lambda_{2}}{4} \delta_{a b} \delta_{c d} .
\end{aligned}
$$

In the Hartree approximation, $V_{2}$ does not explicitly depend on $\alpha$, either are functions of $\alpha$.

The stationary conditions for the condensates $\bar{\sigma}_{0}$ and $\bar{\sigma}_{8}$ are then written as

$$
\begin{aligned}
c \sqrt{\frac{2}{3}}\left(2 m_{l}+m_{s}\right)= & \bar{\sigma}_{0}\left[\mu^{2}+\frac{\lambda_{1}}{3}\left(\bar{\sigma}_{0}\right)^{2}+\lambda_{2}\left(\bar{\sigma}_{0}\right)^{2}-\frac{B}{\sqrt{6}} \bar{\sigma}_{0}\right]+\left(\bar{\sigma}_{8}\right)^{2}\left[\lambda_{1} \bar{\sigma}_{0}+\lambda_{2} \bar{\sigma}_{0}-\frac{\lambda_{1}}{3 \sqrt{2}} \bar{\sigma}_{8}+\frac{B}{2 \sqrt{6}}\right] \\
& -k c\left[\frac{m_{l}}{3}\left(8 \bar{\sigma}_{0}-2 \sqrt{2} \bar{\sigma}_{8}\right)+\frac{m_{s}}{3}\left(4 \bar{\sigma}_{0}+2 \sqrt{2} \bar{\sigma}_{8}\right)\right] \\
& -3 G_{0 b c}\left(\int_{k} \overline{\mathcal{S}}_{c b}(k)-\int_{k} \overline{\mathcal{P}}_{c b}(k)\right)+4 F_{0 b c d} \bar{\sigma}_{d} \int_{k} \overline{\mathcal{S}}_{c b}(k)+4 H_{0 b c d} \bar{\sigma}_{d} \int_{k} \overline{\mathcal{P}}_{c b}(k), \\
c \frac{2}{\sqrt{3}}\left(m_{l}-m_{s}\right)= & \bar{\sigma}_{8}\left[\mu^{2}+\frac{B}{\sqrt{6}} \bar{\sigma}_{0}+\frac{B}{2 \sqrt{3}} \bar{\sigma}_{8}+\left(\lambda_{1}+\lambda_{2}\right) \bar{\sigma}_{0}^{2}-\frac{\lambda_{1}}{\sqrt{2}} \bar{\sigma}_{0} \bar{\sigma}_{8}+\left(\frac{\lambda_{1}}{2}+\lambda_{2}\right)\left(\bar{\sigma}_{8}\right)^{2}\right] \\
& -k c\left[\frac{m_{l}}{3}\left(-2 \sqrt{2} \bar{\sigma}_{0}-8 \bar{\sigma}_{8}\right)+\frac{m_{s}}{3}\left(2 \sqrt{2} \bar{\sigma}_{0}+2 \bar{\sigma}_{8}\right)\right] \\
& -3 G_{8 b c}\left(\int_{k} \overline{\mathcal{S}}_{c b}(k)-\int_{k} \overline{\mathcal{P}}_{c b}(k)\right)+4 F_{8 b c d} \bar{\sigma}_{d} \int_{k} \overline{\mathcal{S}}_{c b}(k)+4 H_{8 b c d} \bar{\sigma}_{d} \int_{k} \overline{\mathcal{P}}_{c b}(k),
\end{aligned}
$$

where

$$
G_{a b c}=\frac{B}{6}\left[d_{a b c}-\frac{3}{2}\left(\delta_{a 0} d_{0 b c}+\delta_{b 0} d_{a 0 c}+\delta_{c 0} d_{a b 0}\right)+\frac{9}{2} d_{000} \delta_{a 0} \delta_{b 0} \delta_{c 0}\right]
$$

In the Hartree approximation, the self-energies $\Sigma_{a b}$ and $\Pi_{a b}$ included in the dressed propagators $\overline{\mathcal{S}}_{a b}$ and $\overline{\mathcal{P}}_{a b}$ [as in Eqs. (3.5)] are independent of momenta. To solve the stationary conditions for $\bar{\sigma}, \overline{\mathcal{S}}$ and $\overline{\mathcal{P}}$ in Eq. (3.4), we may therefore take an ansatz for the dressed propagator like 


$$
\begin{aligned}
& \overline{\mathcal{S}}_{a b}^{-1}(k)=-k^{2} \delta_{a b}+\left(M_{S}^{2}\right)_{a b}, \\
& \overline{\mathcal{P}}_{a b}^{-1}(k)=-k^{2} \delta_{a b}+\left(M_{P}^{2}\right)_{a b} .
\end{aligned}
$$

where $\left(M_{S}\right)_{a b}$ and $\left(M_{P}\right)_{a b}$ are dressed scalar and pseudoscalar masses in the matrix form, which are evaluated from Eqs. (3.5) and (3.6) as

$\left[M_{S}^{2}\right]_{a b}=\left[m_{S}^{2}(\bar{\sigma})\right]_{a b}+4 F_{a b c d} \int_{k} \overline{\mathcal{S}}_{c d}(k)+4 H_{a b c d} \int_{k} \overline{\mathcal{P}}_{c d}(k)$,
$\left[M_{P}^{2}\right]_{a b}=\left[m_{P}^{2}(\bar{\sigma})\right]_{a b}+4 F_{a b c d} \int_{k} \overline{\mathcal{P}}_{c d}(k)+4 H_{a b c d} \int_{k} \overline{\mathcal{S}}_{c d}(k)$.

Note also that in the Hartree approximation, only the real part of the mass matrices are generated. The dressed-mass matrices can be diagonalized by orthogonal transformations,

$$
\left[\tilde{M}_{S, P}^{2}\right]_{(i)} \delta_{i j}=\left(O_{S, P}^{\prime-1}\right)_{i a}\left[M_{S, P}^{2}\right]_{a b}\left(O_{S, P}^{\prime}\right)_{b j},
$$

where $\left(\tilde{M}_{S, P}^{2}\right)_{(i)}$ are mass eigenvalues for the dressedmasses. Note that in general the transformation matrices $O_{S, P}^{\prime}$ are different from those at the tree-level [Eqs. (2.18) and (2.22)], due to the induced temperature dependence on the mixing angles $\theta_{S, P}$, so the mixing angles are computed as follows:

$$
\theta_{S, P}=\frac{1}{2} \arctan \left[\frac{2\left(M_{S, P}^{2}\right)_{08}}{\left(M_{S, P}^{2}\right)_{00}-\left(M_{S, P}^{2}\right)_{88}}\right] .
$$

By using the transformation matrix $O_{S, P}^{\prime}$ in Eq. (3.13), the dressed-propagators for $\overline{\mathcal{S}}$ and $\overline{\mathcal{P}}$ can be diagonalized as

$$
\begin{aligned}
& \tilde{\mathcal{S}}_{(i)}(k) \delta_{i j}=\left(O^{\prime-1}\right)_{i a} \overline{\mathcal{S}}_{a b}(k)\left(O_{S}^{\prime}\right)_{b j}, \\
& \tilde{\mathcal{P}}_{(i)}(k) \delta_{i j}=\left(O_{P}^{\prime-1}\right)_{i a} \overline{\mathcal{P}}_{a b}(k)\left(O_{P}^{\prime}\right)_{b j},
\end{aligned}
$$

where $\tilde{\mathcal{S}}_{(i)}(k)$ and $\tilde{\mathcal{P}}_{(i)}(k)$ are the dressed propagators in the mass eigenbasis.

The loop integral for the dressed propagators in Eq. (3.15) consists of the vacuum-contribution part (zero-temperature part) plus the thermal-correction part. Although the vacuum part has the ultraviolet (UV) divergences and is subject to renormalization schemes, the thermal contribution to the loop integral is independent of UV divergence and renormalization. Actually, the qualitative results obtained based on the CJT analysis at finite temperatures are fairly insensitive to renormalization schemes, as was studied in [28-30]. Thus, as far as qualitative features deduced from nonperturbative corrections are concerned, we may be almost free from the UV sensitivity, allowing to just work on the thermal corrections from the meson loops. Then the dressed propagators $\tilde{\mathcal{S}}_{(i)}(k)$ and $\tilde{\mathcal{P}}_{(i)}(k)$ are evaluated by only including the thermalmeson loop terms as

$$
\begin{aligned}
\int_{k} \tilde{\mathcal{S}}_{(i)}(k) & =\int \frac{d^{3} \boldsymbol{k}}{(2 \pi)^{3}} \frac{1}{\epsilon_{\boldsymbol{k}}\left[\left(\tilde{M}_{S}^{2}\right)_{(i)}\right]}\left(\exp \left\{\frac{\epsilon_{\boldsymbol{k}}\left[\left(\tilde{M}_{S}^{2}\right)_{(i)}\right]}{T}\right\}-1\right)^{-1}, \\
\int_{k} \tilde{\mathcal{P}}_{(i)}(k) & =\int \frac{d^{3} \boldsymbol{k}}{(2 \pi)^{3}} \frac{1}{\epsilon_{\boldsymbol{k}}\left[\left(\tilde{M}_{P}^{2}\right)_{(i)}\right]}\left(\exp \left\{\frac{\epsilon_{\boldsymbol{k}}\left[\left(\tilde{M}_{P}^{2}\right)_{(i)}\right]}{T}\right\}-1\right)^{-1},
\end{aligned}
$$

where the $\epsilon_{k}$ represents the relativistic energy of the mesons with momentum $\boldsymbol{k}, \epsilon_{\boldsymbol{k}}\left[\left(\tilde{M}_{S(P)}^{2}\right)_{(i)}\right]=\sqrt{\boldsymbol{k}^{2}+\left(\tilde{M}_{S(P)}^{2}\right)_{(i)}}$.

The thermal-dependent quark condensates, $\langle\bar{l} l\rangle(T)$ and $\langle\bar{s} s\rangle(T)$, are evaluated as functions of the dressed propagators, and calculated as follows:

$$
\begin{aligned}
\langle\bar{l} l\rangle(T)= & \left.\frac{\partial V_{\mathrm{eff}}[\alpha, S, P]}{\partial m_{l}}\right|_{\alpha=\bar{\sigma}, S=\overline{\mathcal{S}}, P=\overline{\mathcal{P}}} \\
= & \left.\frac{\partial V(\alpha)}{\partial m_{l}}\right|_{\alpha=\bar{\sigma}}+\frac{1}{2} \int_{k}\left[\frac{\partial\left[m_{S}^{2}(\alpha)\right]_{a b}}{\partial m_{l}} S_{b a}(k)\right. \\
& \left.+\frac{\partial\left[m_{P}^{2}(\alpha)\right]_{a b}}{\partial m_{l}} P_{b a}(k)\right]\left.\right|_{\alpha=\bar{\sigma}, S=\overline{\mathcal{S}}, P=\overline{\mathcal{P}}} \\
= & -2 c\left(\bar{\Phi}_{1}(T)+2 k \bar{\Phi}_{1}(T) \bar{\Phi}_{3}(T)\right)+\cdots, \\
\langle\bar{s} S\rangle(T)= & \left.\frac{\partial V_{\mathrm{eff}}[\alpha, S, P]}{\partial m_{s}}\right|_{\alpha=\bar{\sigma}, S=\overline{\mathcal{S}}, P=\overline{\mathcal{P}}} \\
= & \left.\frac{\partial V(\alpha)}{\partial m_{l}}\right|_{\alpha=\bar{\sigma}}+\frac{1}{2} \int_{k}\left[\frac{\partial\left[m_{S}^{2}(\alpha)\right]_{a b}}{\partial m_{s}} S_{b a}(k)\right. \\
& \left.+\frac{\partial\left[m_{P}^{2}(\alpha)\right]_{a b}}{\partial m_{s}} P_{b a}(k)\right]\left.\right|_{\alpha=\bar{\sigma}, S=\overline{\mathcal{S}}, P=\overline{\mathcal{P}}} \\
= & -2 c\left(\bar{\Phi}_{3}(T)+2 k \bar{\Phi}_{1}^{2}(T)\right)+\cdots,
\end{aligned}
$$

where the thermal-meson loop corrections for quark condensates are included in terms denoted as “...", where are, in terms of the quark mass expansion, suppressed compared with the leading order terms in Eq. (3.17). In numerically estimating the temperature dependence of the quark condensates, we will pick up only the leading terms in Eq. (3.17). Since the $\bar{\Phi}_{1}$ and $\bar{\Phi}_{3}$ are determined by solving the stationary conditions in Eqs. (3.9) and (3.12), so that the quark condensates nonperturbatively get thermal effects. Of interest is that the quark condensates explicitly have a remnant of the axial-anomaly induced-flavor breaking, what we call the $k$-term, $V_{\mathrm{SB}-a n o m}$, as seen in Eq. (3.17). Thus, the axial-anomaly induced-flavor breaking would be expected to directly contribute to the chiral restoration phenomena at finite temperatures. 


\section{NUMERICAL ANALYSIS ON NONPERTURBATIVE FLAVOR BREAKING AT CHIRAL CROSSOVER CRITICALITY}

We are now ready to study how the nonperturbative flavor breaking can be generated at finite temperatures across the chiral critical phenomena, such as the chiral crossover. To this end, we take the following values for the model parameters as inputs

$$
\begin{array}{rlrl}
\mu^{2} & =1.02 \times 10^{4} \mathrm{MeV}^{2}, & \lambda_{1}=11.8, \quad \lambda_{2}=20.4, \\
c m_{l} & =6.11 \times 10^{5} \mathrm{MeV}^{3}, & c m_{s}=198 \times 10^{5} \mathrm{MeV}^{3} \\
B & =3.85 \times 10^{3} \mathrm{MeV}, \quad k=3.40 \mathrm{GeV}^{-1}, \quad
\end{array}
$$

with which the present model at vacuum well reproduces the scalar and pseudoscalar meson spectra, including the inverse mass hierarchy for scalar mesons ligher than $1 \mathrm{GeV}$ [11]: From Eqs. (2.17) and (2.21) with the input parameters as above, the meson masses in the vacuum (at zero temperature) are estimated to be

$$
\begin{aligned}
m\left[f_{0}(500)\right] & =672.4 \mathrm{MeV}, & & m\left[f_{0}(980)\right]=990.4 \mathrm{MeV}, \\
m_{a_{0}} & =937.6 \mathrm{MeV}, & & m_{\kappa}=863.4 \mathrm{MeV}, \\
m_{\eta^{\prime}} & =958.2 \mathrm{MeV}, & & m_{\eta}=552.9 \mathrm{MeV}, \\
m_{\pi} & =137.9 \mathrm{MeV}, & & m_{K}=494.1 \mathrm{MeV},
\end{aligned}
$$

which indeed shows good agreement with the experimental values [31].

In addition, we predict the topological susceptibility to be

$$
\begin{aligned}
\chi_{\text {top }}(T=0) & \simeq 0.0263 / \mathrm{fm}^{4} \quad \text { or } \\
\left(\chi_{\text {top }}(T=0)\right)^{1 / 4} & \simeq 79.4 \mathrm{MeV} .
\end{aligned}
$$

Actually, the recent lattice QCD data, with $2+1(+1)$ flavors having a physical pion mass and the continuum limit being taken, predicts the topological susceptibility at the zero temperature as $\chi_{\text {top }}(T=0)=0.019(9) / \mathrm{fm}^{4}[32]$, and $\chi_{\text {top }}(T=0)=0.0245(24)_{\text {stat }}(03)_{\text {flow }}(12)_{\text {cont }} / \mathrm{fm}^{4}[33]$. For the latter the first error is statistical, the second error is systematic error and the third error comes from changing the upper limit of the lattice spacing range in the fit. On the lattice, the topological susceptibility is defined through a certain smoothing process to reduce UV sensitivity of the topological charge operator, which brings a systematic error. Besides, sampling among different topological sectors is a nontrivial task for lattice QCD simulations [34-38]. Although their central values do not agree with each other, from a conservative point of view, we may say that the difference between them is interpreted as a systematic error from the individual lattice QCD calculation.

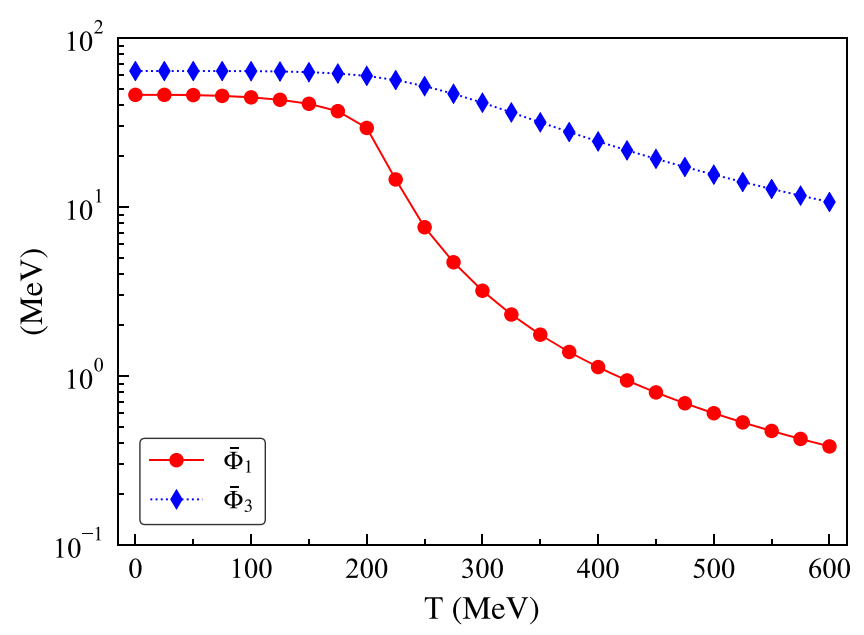

FIG. 1. The $\bar{\Phi}_{1}$ and $\bar{\Phi}_{3}$ as a function of the temperature.

\section{A. Chiral order parameters}

In Fig. 1, we first show a plot on the scalar condensates $\bar{\Phi}_{1,3}$ as a function of temperature, which are evaluated by solving the stationary conditions for the CJT effective potential in Eq. (3.4). ${ }^{3}$ The figure tells us that the $\bar{\Phi}_{1}$ becomes smaller more rapidly than the $\bar{\Phi}_{3}$ does. This tendency implies that the two-flavor chiral symmetry, controlled by the $\Phi_{1}$, can be restored faster than that for the heavier strange quark, by the $\Phi_{3}$. However, it looks like that the chiral symmetry is not exactly restored, so it may be sort of crossover phenomenon. The similar scaling property of scalar condensates with respect to the temperature has been observed in other three-flavor models based on the CJT formalism $[28,29]$, in which the axial-anomaly induced-flavor breaking, the $k$-term in $V_{\mathrm{SB} \text {-anom, }}$, is not incorporated.

We next discuss thermal effects on the quark condensates $\langle\bar{q} q\rangle(T)$ in Eq. (3.17). Figure 2 displays the temperature dependence of the light quark condensate $\langle\bar{l} l\rangle(T) /\langle\bar{l} l\rangle(T=0)$. The figure shows that the light quark condensate does not exactly reach zero, though being monotonically damping as $T$ gets larger. This implies that the chiral phase transition does not happen, instead, the theory undergoes a chiral crossover, as was indicated in the scaling of scalar condensates above. This crossover phenomenon is in a qualitative sense consistent with the current result of the lattice QCD with $2+1$ flavors [1-5]. It it interesting to note that the chiral crossover has been observed even in the absence of the axial-anomaly inducedflavor breaking term $V_{\mathrm{SB} \text {-anom }}$, so, in this sense, the $k$ term is irrelevant to realize the chiral crossover.

\footnotetext{
${ }^{3}$ At the vacuum $T=0$, the scalar condensates are well degenerated, $\bar{\Phi}_{3}(T=0) / \bar{\Phi}_{1}(T=0) \simeq 1.38$. It indicates that the three-flavor linear sigma model approximately has the $S U(3)$-flavor symmetry at the vacuum.
} 


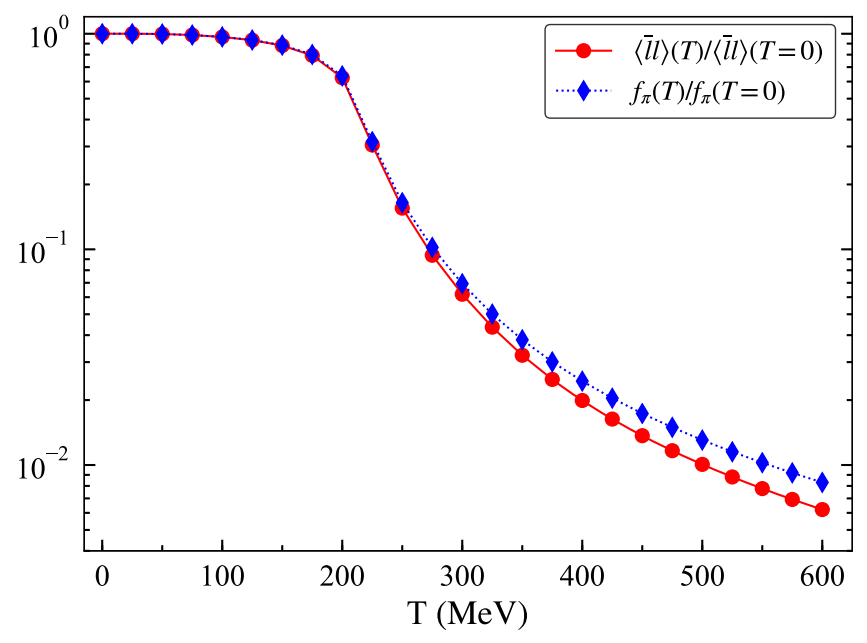

FIG. 2. The temperature dependence of the light quark condensates and the pion decay constant, normalized to those vacuum values.

From Fig. 2, we may estimate the pseudocritical temperature of the chiral crossover, simply by identifying it as a temperature $T_{p c}^{*}$, where $d^{2}\langle\bar{l} l\rangle(T) /\left.d T^{2}\right|_{T=T_{p c}^{*}}=0$. We then find $T_{p c}^{*} \simeq 215 \mathrm{MeV}$. Note that the definition of our $T_{p c}^{*}$ is different from the lattice QCD's yielding the pseudocritical temperature $T_{p c} \simeq 155 \mathrm{MeV}$ and has been estimated to be larger [1-5]: By construction, the present chiral effective model can only include operators with single current quark mass matrix $\mathcal{M}$, so we cannot evaluate the chiral susceptibility, through which the pseudocritical temperature in the lattice simulation is defined. However the temperature at which the maximum of the chiral susceptibility should reach is usually compatible with the inflection point of the chiral condensate in lattice QCD data, i.e., the point where $d^{2}\langle\bar{l} l\rangle(T) /\left.d T^{2}\right|_{T=T_{p c}}=0$. So, we may naively have quantitative comparison between $T_{p c}$ and $T_{p c}^{*}$, to find about $30 \%$ deviation. We will come back to this quantitative discrepancy in the present analysis, in the later section.

To make manifest how the axial-anomaly induced-flavor breaking affects the chiral crossover phenomenon, we compare the ratio $\langle\bar{l} l\rangle(T) /\langle\bar{l} l\rangle(T=0)$ with the pion decay constant normalized to the vacuum value $f_{\pi}(T) / f_{\pi}(T=0)$, which corresponds to the temperature dependence of the light quark condensates in the absence of the axialanomaly induced-flavor breaking term. Figure 2 shows that the $\langle\bar{l} l\rangle(T) /\langle\bar{l} l\rangle(T)$ drops somewhat rapidly than the $f_{\pi}(T) / f_{\pi}(T=0)$. This implies that the anomaly-induced flavor breaking acts as a catalyzer toward the chiral restoration.

The chiral condensate has the UV divergence and is need to be renormalized to get the finite quantity. To eliminate the quadratic divergences tagged with the quark mass in the chiral condensates, we use the subtracted chiral condensate $\Delta_{l, s}(T)=\langle\bar{l} l\rangle-\frac{2 m_{l}}{m_{s}}\langle\bar{s} s\rangle$ as the order parameter of the chiral phase transition: Fig. 3 shows the temperature

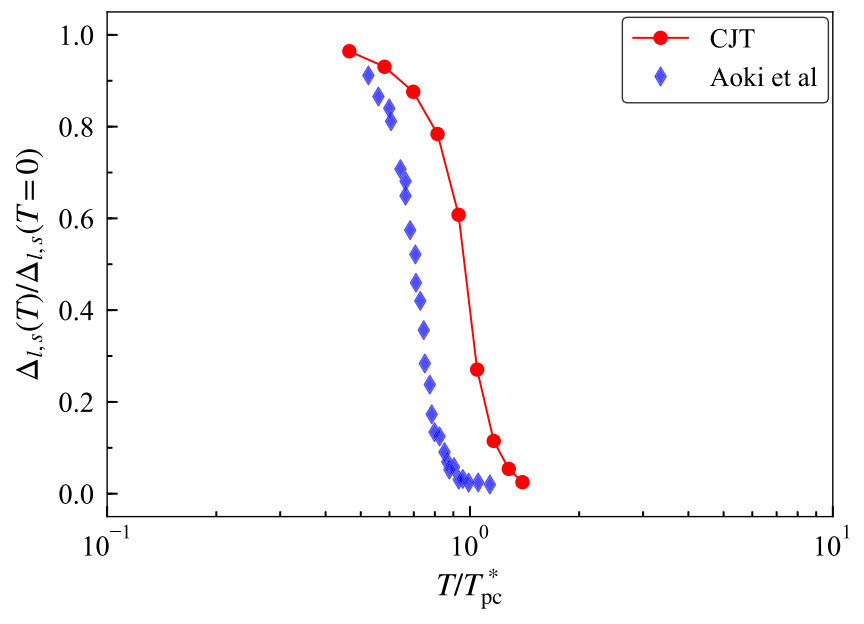

FIG. 3. The comparison of the subtracted chiral condensate $\Delta_{l, s}(T)$ with the lattice QCD data [39].

dependence of the subtracted chiral condensate based on the CJT analysis, in comparison with the lattice QCD observation [39]. Although the deviation from the lattice QCD data [39] is read as about 30\% around the pseudocritical temperature regions $T \simeq T_{p c}^{*}$, the CJT analysis qualitatively supplies the chiral crossover as observed in the lattice QCD observation [39].

We now show the significance of the flavor breaking between the light and strange quark condensates at around the chiral crossover. Note again that quark condensates themselves potentially include UV divergences, hence dependence on the renormalization scales, so would be unreasonable to merely compare those for light and strange flavors, in light of the lattice observation. In Fig. 4 we thus plot the renormalization-scale invariant combinations $m_{s}\langle\bar{s} s\rangle(T)$ and $m_{l}\langle\bar{l} l\rangle(T)$ normalized to $f_{\pi}^{4}$ [panel (a)] and its ratio $m_{s}\langle\bar{s} s\rangle(T) / m_{l}\langle\bar{l} l\rangle(T)$ [panel (b)], as a function of the temperature. At the zero temperature, the ratio is computed as $m_{s}\langle\bar{s} s\rangle(T=0) / m_{l}\langle\bar{l} l\rangle(T=0) \simeq$ $(32.4) \times(1.18) \simeq 38$ (with $\left.m_{s} / m_{l} \simeq 32.4\right)$, which is in good agreement with the recent lattice simulation (at a physical pion mass and the renormalization scale of $2 \mathrm{GeV}$ ), $m_{s}\langle\bar{s} s\rangle(T=0) / m_{l}\langle\bar{l} l\rangle(T=0)=(32.4) \times(1.08 \pm 0.16) \quad$ [7] Therefore, the $S U(3)_{V}$ flavor symmetry between the light quark and the strange quark condensate is approximately preserved at the zero temperature. Moving on to the finite temperature environment, the ratio $m_{s}\langle\bar{s} s\rangle(T) / m_{l}\langle\bar{l} l\rangle(T)$ is drastically enhanced and monotonically increased around the pseudocritical temperature $T_{p c}^{*} \simeq 215 \mathrm{MeV}$, so that the violation of flavor symmetry between the light quark and the strange quark condensates gets more eminent over the chiral criticality [10]. This dramatic enhancement has been also observed in several analyses on hot lattice QCD [8,9]. Furthermore, to extract the contribution of $V_{\mathrm{SB}-\text { anom }}$ to the flavor breaking, we compare the $m_{s}\langle\bar{s} s\rangle(T) / m_{l}\langle\bar{l} l\rangle(T)$ with the $m_{s} \bar{\Phi}_{3}(T) / m_{l} \bar{\Phi}_{1}(T)$ which is identical to the one 


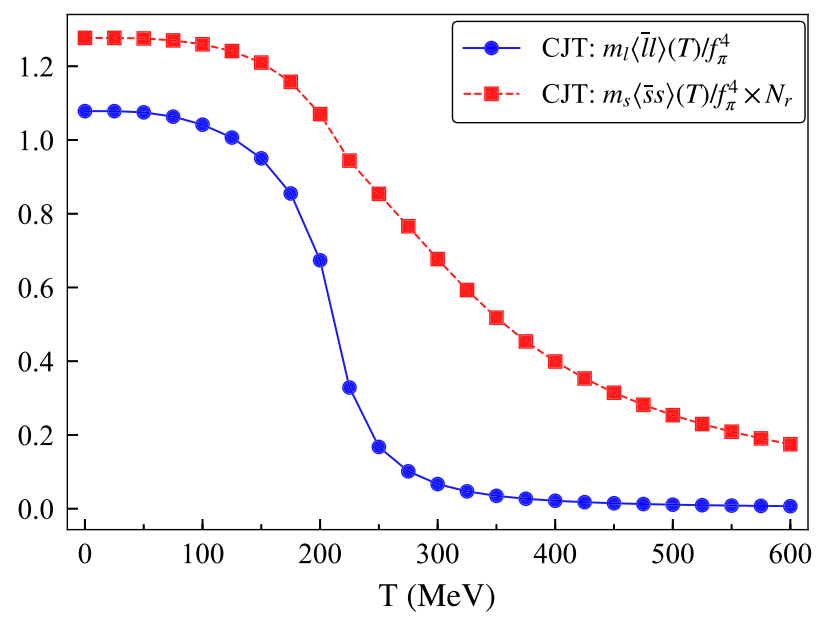

(a)

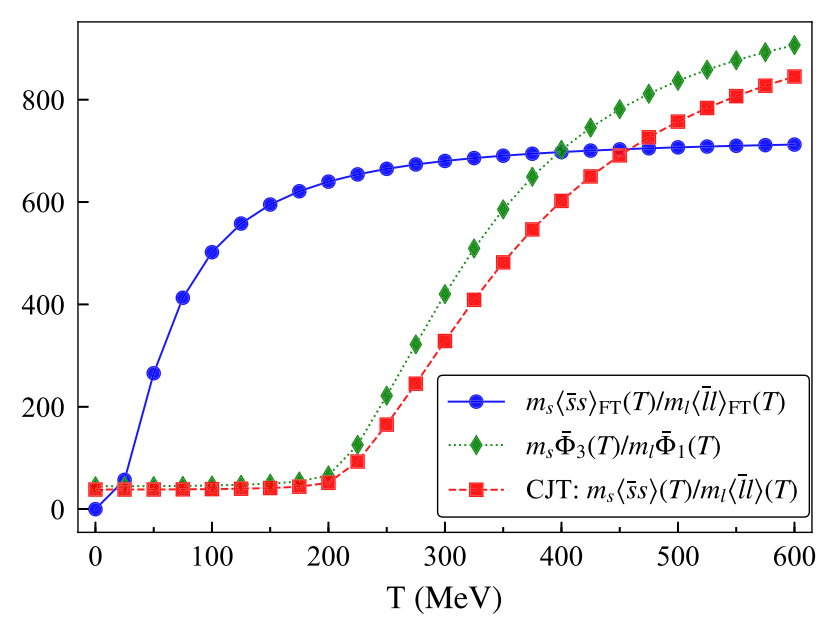

(b)

FIG. 4. Plots on the temperature dependence of the renormalization-scale invariant quark-condensates $m_{s}\langle\bar{s} s\rangle(T)$ and $m_{l}\langle\bar{l} l\rangle(T)$ normalized to $f_{\pi}^{4}$, individually in panel (a) and via its ratio together with comparison with $m_{s} \bar{\Phi}_{3}(T) / m_{l} \bar{\Phi}_{1}(T)$ and $m_{s}\langle\bar{s} s\rangle_{\mathrm{FT}}(T) / m_{l}\langle\bar{l} l\rangle_{\mathrm{FT}}(T)$ obtained in the free theory of quarks, in panel (b). In panel (a) the $m_{s}\langle\bar{s} s\rangle(T) / f_{\pi}^{4}$ has been multiplied by a factor of $N_{r}=1 / 32.4$, corresponding to the value of $m_{l} / m_{s}$ estimated from the present linear sigma model.

without the anomaly-induced flavor breaking effect. Figure 4 shows that the axial-anomaly induced-flavor breaking term plays a role to reduce the magnitude of the flavor breaking.

To clarify that the flavor breaking certainly comes from the nonperturbative thermal contribution, in Fig. 4 we also compare the $m_{s}\langle\bar{s} s\rangle(T) / m_{l}\langle\bar{l} l\rangle(T)$ with the one corresponding to the free theory (FT) of quarks, in which the quarks behave non-interacting free-particles. The ratio of free-quark condensates $m_{s}\langle\bar{s} s\rangle_{\mathrm{FT}}(T) / m_{l}\langle\bar{l} l\rangle_{\mathrm{FT}}(T)$ is perturbatively obtained from the thermal one-loop calculation. We provide the detailed expression of quark condensates in the FT in the Appendix B. For the low temperature regions where $T<m_{s} \sim 100 \mathrm{MeV}$, the FT-quark condensate ratio $m_{s}\langle\bar{s} s\rangle_{\mathrm{FT}}(T) / m_{l}\langle\bar{l} l\rangle_{\mathrm{FT}}(T)$ keeps the value below $\left(m_{s} / m_{l}\right)^{2} \simeq(27)^{2}=729$, because of the naive Boltzmann suppression for the strange quark contribution. After passing $T \simeq m_{s} \sim 100 \mathrm{MeV}$, the $m_{s}\langle\bar{s} s\rangle_{\mathrm{FT}}(T) / m_{l}\langle\bar{l} l\rangle_{\mathrm{FT}}(T)$ asymptotically approaches $\left(m_{s} / m_{l}\right)^{2}=729$, which merely reflects the trivial and overall flavor-breaking just by quark masses: $m_{q}\langle\bar{q} q\rangle \sim m_{q}^{2} T^{2}$. In contrast, in whole low-temperature regions $T<T_{p c}^{*} \simeq 215 \mathrm{MeV}$ the $\mathrm{CJT}$ analysis exhibits a gigantic suppression for the quark-condensate ratio more than the Boltzmann suppression, to respect the flavor symmetry, i.e., the vacuum value $\sim\left(m_{s} / m_{l}\right) \times 1$. This flavor symmetric behavior is consistent with the conventional three-flavor ChPT observation, in which the vectorial $S U(3)$ flavor symmetry cannot explicitly be violated at the leading order of the chiral expansion based on the nonlinear-sigma model setup, so that $m_{s}\langle\bar{s} s\rangle_{\mathrm{ChPT}}(T) / m_{l}\langle\bar{l} l\rangle_{\mathrm{ChPT}}(T) \simeq\left(m_{s} / m_{l}\right) \times 1$ even including the next-to leading order corrections. As the temperature further increases, the CJT analysis nonperturbatively undergoes the chiral crossover around the pseudocritical temperature $T_{p c}^{*} \simeq 215 \mathrm{MeV}$ (see Figs. 1, 2 and panel (a) of 4), where the light quark condensate $m_{l}\langle\bar{l} l\rangle(T)$ starts to drop more efficiently than the strange quark condensate $m_{s}\langle\bar{s} s\rangle(T)$. Consequently, the quark-condensate ratio rapidly starts to grow from $T \simeq T_{p c}^{*}$. This is a nontrivial flavor breaking, essentially different from the trivial FT flavor violation just by $m_{s} / m_{l}$. In the end, at around $T \simeq 600 \mathrm{MeV}$, the quark-condensate ratio asymptotically merges with the FT yielding the trivial-flavor breaking value $\left(m_{s} / m_{l}\right)^{2}$. This would imply that the present linear sigma model would converge to an ideal-quark gas picture consistently with the asymptotic free nature of the underlying QCD. Thus we can conclude that what we call a nonperturbative flavor breaking is certainly a nonperturbative output in association with the characteristic chiral crossover phenomenon.

\section{B. Meson spectral properties}

The feature of the chiral crossover criticality should be reflected in the meson dynamics. In particular, the mixing of singlet and octet states are strongly correlated with the chiral symmetry breaking. In this subsection, we first discuss the mixing structure of constituents in the $f_{0}(500)$ and $f_{0}(980) \quad\left(\eta^{\prime}\right.$ and $\left.\eta\right)$ via the temperature dependent mixing angles $\theta_{S, P}$ in Eq. (3.14), across the crossover criticality.

Figure 5 shows the $\theta_{S, P}$ as a function of temperature. When the temperature is increased up to around the pseudocritical temperature $T_{p c}^{*} \simeq 215 \mathrm{MeV}$, the $\theta_{S}$ becomes smaller. On the other hand, the $\theta_{P}$ dramatically grows. After arriving at around $T_{p c}^{*}$, the mixing angles $\theta_{S, P}$ converge gradually to the ideal mixing angle $\theta_{S, P}=\arcsin (1 / \sqrt{3}) \simeq 35.3^{\circ}$, where the mass eigenstates completely separate into the strange-quark 


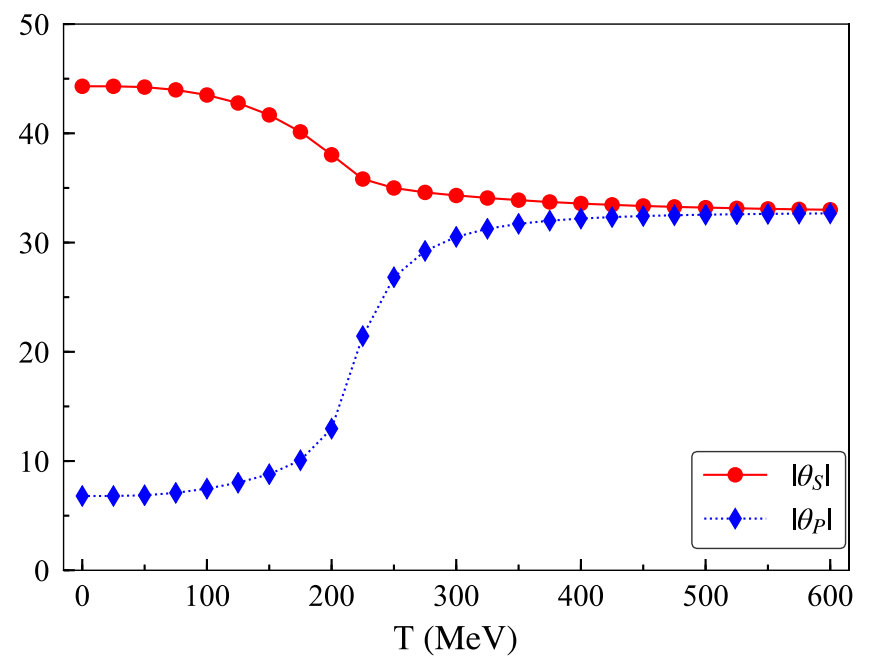

FIG. 5. The mixing angles of the scalar $\left(\sigma_{0}, \sigma_{8}\right)$ and the pseudoscalars $\left(\pi_{0}, \pi_{8}\right)$ as a function of temperature.

and nonstrange quark scalars. ${ }^{4}$ Therefore, this implies that above $T_{p c}^{*}$ the $f_{0}(500)$ and $\eta^{\prime}$ mesons are identified as light-quark $(\bar{l} \bar{l})$ scalar sates, and the $f_{0}(980)$ and $\eta$ mesons as $s \bar{s}$ states. Actually, other three-flavor model analyses based on the CJT formalism [28] have also predicted the same critical phenomenon for the mixing structure, where the anomaly-related flavor breaking term $V_{\mathrm{SB} \text {-anom }}$ is not involved. In that sense, we may conclude that the axialanomaly induced-flavor breaking is not essential so much for the meson mixing structure in relation to the critical phenomenon.

Second, we focus on the criticality effect on the meson masses. It turns out that a couple of interesting consequences of the chiral crossover emerge in meson spectroscopy. In Fig. 6, we show the temperature dependence of the dressed masses for scalar mesons and the pseudoscalar mesons. When the temperature is increased up to around the pseudocritical temperature $T_{p c}^{*}$, the dressed-scalar masses drop. In particular, the dropping rate for the $f_{0}(500)$ meson gets prominent. As for the pseudoscalar masses, the dressed mass of $\eta^{\prime}$ slightly decreases below the pseudocritical temperature $T_{p c}^{*}$, while the other pseudoscalar masses grow. Above $T_{p c}^{*}$, especially at $T \geq 300 \mathrm{MeV}$, all of the dressed-scalar and -pseudoscalar masses monotonically increase and tend to degenerate. We shall discuss more details on these scaling properties below.

It would be interesting to compare the thermal masses in Fig. 6 with the screening masses observed on the lattice QCD [40]. We find that the mass scaling properties of the

\footnotetext{
${ }^{4}$ Indeed, at higher temperatures around $T \simeq 600 \mathrm{MeV}$, where the ideal mixing is almost perfectly realized, the $f_{0}(500)$ and $\eta^{\prime}$ meson are completely made of the fluctuating modes from $\bar{\Phi}_{1}$ (nonstrange, or light-quark components), while the $f_{0}(980)$ and the $\eta$ meson are the fluctuating modes from $\bar{\Phi}_{3}$ (strange-quark components).
}

pseudoscalars qualitatively agree with the lattice data. Regarding the scalar mesons, accurate lattice studies on identifying the bound state channels currently involve some complicated issues. Therefore, direct comparison to our predictions is not straightforward, which would be possibly resolved by the future studies.

As was clarified in [11], we first recall that the axialanomaly induced flavor breaking serves as an important source to realize the inverse mass hierarchy for scalar mesons below $1 \mathrm{GeV}, \quad m\left[f_{0}(980)\right]>m\left[a_{0}(980)\right]>$ $m\left[K_{0}^{*}(700)\right]>m\left[f_{0}(500)\right]$. To examine the thermal effect on the inverse mass hierarchy, we plot the mass differences among scalar mesons in the left panel of Fig. 7. We can see that the mass difference between $m\left[a_{0}(980)\right]$ and $m\left[K_{0}^{*}(700)\right]$ keeps the inverse mass hierarchy, $m\left[a_{0}(980)\right]>$ $m\left[K_{0}^{*}(700)\right]$, even at high temperatures. This implies that the axial-anomaly induced-flavor breaking survives even at high temperatures, above the pseudocritical temperature, and continues to keep the mass difference as in vacuum. This surviving flavor breaking in the mass difference $m\left[a_{0}(980)\right]-m\left[K_{0}^{*}(700)\right]$ might be a crucial signal to indirectly detect the anomaly-induced flavor breaking at hot QCD, maybe, at lattice QCD in the future.

Furthermore, in the left panel of Fig. 7 one can find that the order between $m\left[f_{0}(980)\right]$ and $m\left[a_{0}(980)\right]$ flips around $T_{p c}^{*}$ :

$$
\begin{aligned}
m\left[a_{0}(980)\right] & >m\left[f_{0}(980)\right]>m\left[K_{0}^{*}(700)\right] \\
& >m\left[f_{0}(500)\right] \quad \text { at } T / T_{p c}^{*} \sim 1
\end{aligned}
$$

This hierarchy flipping happens due to nonperturbative thermal-meson loop corrections, which have triggered a new flavor-breaking structure in the scalar meson spectra, and it is worth exploring in lattice simulations in the future.

In the right panel of Fig. 7, we show the mass differences for the chiral partners and the $U(1)_{A}$ partners. As the temperature increases, the mass difference of the chiral partner between $f_{0}(500)$ and pion becomes smaller. Above the $T_{p c}^{*}$, the $f_{0}(500)$ meson becomes approximately degenerate with pion. Recall back that the $f_{0}(500)$ meson is almost an $\bar{l}$ state scalar. Therefore, the degeneracy between $f_{0}(500)$ and pion may imply the restoration signal for the light quark-chiral symmetry. On the other hand, the $\eta$ meson is identified as an $s \bar{s}$ pseudoscalar state, where the strange quark condensate still has a sufficiently large value compared to the $\langle\bar{l} l\rangle$ as displayed in Fig. 4. The consequence of the surviving $\langle\bar{s} s\rangle$ has been also been reflected in the mass difference between $a_{0}(980)$ and $\eta$ : They tend to most slowly merge among the chiral partners and still keep the sizable size $(\gtrsim 100 \mathrm{MeV})$ even when the $f_{0}(500)$ and pion get almost degenerate at around $T_{p c}^{*}$. This is due to the sizable strange quark contribution to both $a_{0}(980)$ (from the $k$ term and nonperturbative thermal loops) and $\eta$ (from 


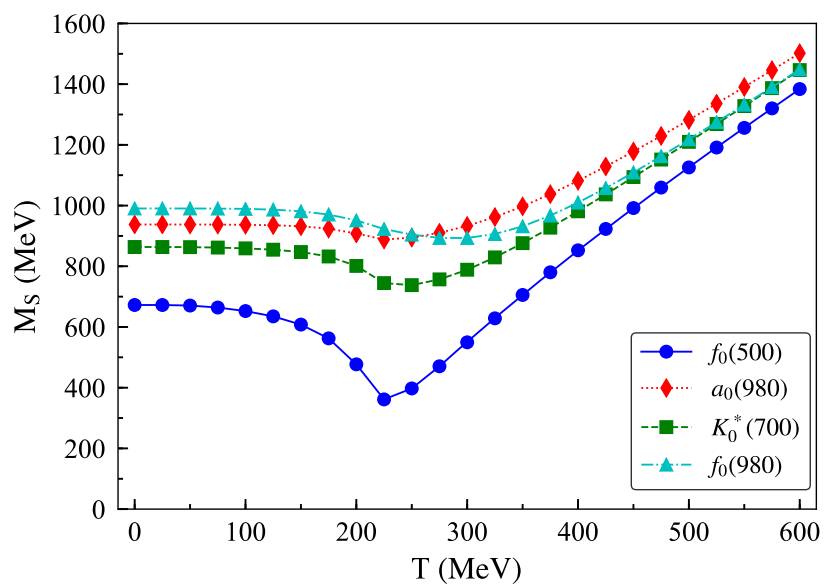

(a)

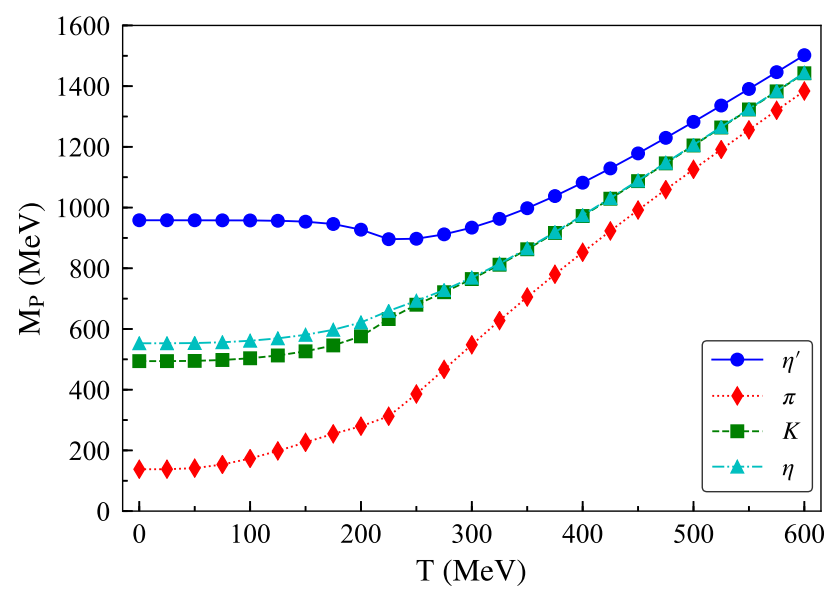

(b)

FIG. 6. The thermal effects on the dressed masses for (a) scalar mesons, and (b) pseudoscalar mesons.

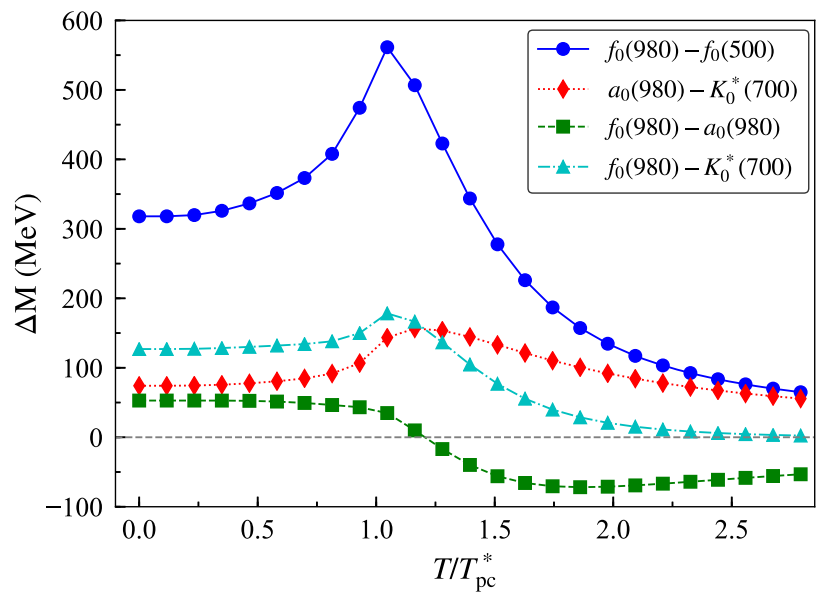

(a)

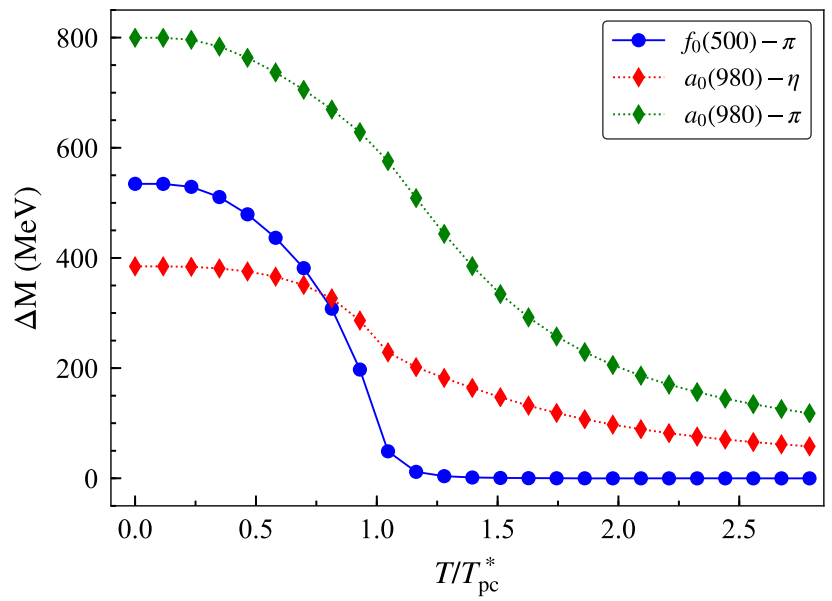

(b)

FIG. 7. Evolution of the mass differences in temperature for (a) scalar mesons, (b) chiral and $U(1)_{A}$ partners.

nonperturbative thermal loops). Thus, the flavor breaking between the light- and strange-quark condensates is certainly reflected in the mass difference of the chiral partners.

Note also (from the green curve in the right panel of Fig. 6) that the mass difference of the $U(1)_{A}$ partner between $a_{0}(980)$ and pion does not become degenerate even at high temperatures enough. This implies that the $U(1)_{A}$ symmetry is still broken even above $T_{p c}^{*}$, in accordance with the surviving flavor breaking induced from the axial anomaly, observed in the scalar meson mass spectra.

The right panel of Fig. 7 also shows the approximate restoration of the $O(4)\left(\simeq S U(2)_{L} \times S U(2)_{R}\right)$ symmetry at high temperatures, which is signaled by the degeneracy of the chiral partner, $f_{0}(500)$ and pion. Of interest is that the $U(1)_{A}$ symmetry detected by the degeneracy of the $U(1)_{A}$ partner is still broken even after the (approximate) $O(4)$ restoration. ${ }^{5}$ This tendency is in agreement with the lattice observation with the physical pion mass [42].

\section{Topological susceptibility}

The generic formula of $\chi_{\text {top }}$ in Eq. (2.42) is still available even at finite temperatures. Using the quark condensates in Eq. (3.17), we evaluate the temperature dependence of the topological susceptibility in Eq. (2.42). What we report

\footnotetext{
${ }^{5}$ In Ref. [41] based on the Ward Identities analysis, it is argued that the $O(4)$ symmetry and the $U(1)_{A}$ symmetry are simultaneously restored by referring to the vanishing condition for the topological susceptibility $\chi_{\text {top }}$, where the flavor breaking effect is not taken into account. So, we may suspect that the contribution of the flavor breaking would cause this discrepancy between our work and the Ward Identities analysis [41] with the flavor symmetry assumed.
} 
here will be summary of the main result in the literature [10], with some discussions compensated.

Some nonperturbative analyses on the QCD topological susceptibility at finite temperatures have so far been done based on chiral effective models [43-46]. However, no discussion on the correlation with quark condensates was made because their topological susceptibilities do not hold the flavor singlet form as in Eq. (2.42) (see also Eq. (2.34), for the flavor singlet condition), hence it seems to have been impossible to find the nonperturbative flavor breaking as addressed in the present paper.

As was shown in Fig. 4, at high temperatures, the flavor symmetry between the light quark condensate and the strange quark condensate is drastically broken. Therefore, according to Eq. (2.42), a similarly significant flavor breaking is expected in the topological susceptibility. Indeed, that takes place, see Fig. 8. The flavor breaking in $\chi_{\text {top }}$ gets larger and larger after reaches the temperature around the chiral crossover, $T_{p c}^{*} \simeq 215 \mathrm{MeV}$, precisely in the same way as the ratio of quark condensates $\left(m_{s}\langle\bar{s} s\rangle(T) / m_{l}\langle\bar{l} l\rangle(T) \gg m_{s} / m_{l}\right)$ follow in Fig. 4.

To extract the strange quark contribution in the topological susceptibility, in Fig. 8 we show the temperature dependence of $\chi_{\text {top }}(T)$ normalized to the one in the threeflavor universal limit, $\chi_{\text {top }}^{3 \mathrm{fl}}=\left(\frac{2}{m_{l}}+\frac{1}{m_{s}}\right)^{-1}\langle\bar{l} l\rangle(T)$. We see that due to the sizable strange quark condensate as seen from Fig. 4, the strange quark contribution to the topological susceptibility is rapidly enhanced from $T \simeq T_{p c}^{*}$. This enhancement is essentially driven by the sizable strange quark condensates acting as a catalyzer for the $U(1)_{A}$ breaking above the pseudocritical temperature. Eventually, after arriving at the high temperature regions where $m_{s}\langle\bar{s} s\rangle(T) / m_{l}\langle\bar{l} l\rangle(T)$ reaches the trivial-flavor

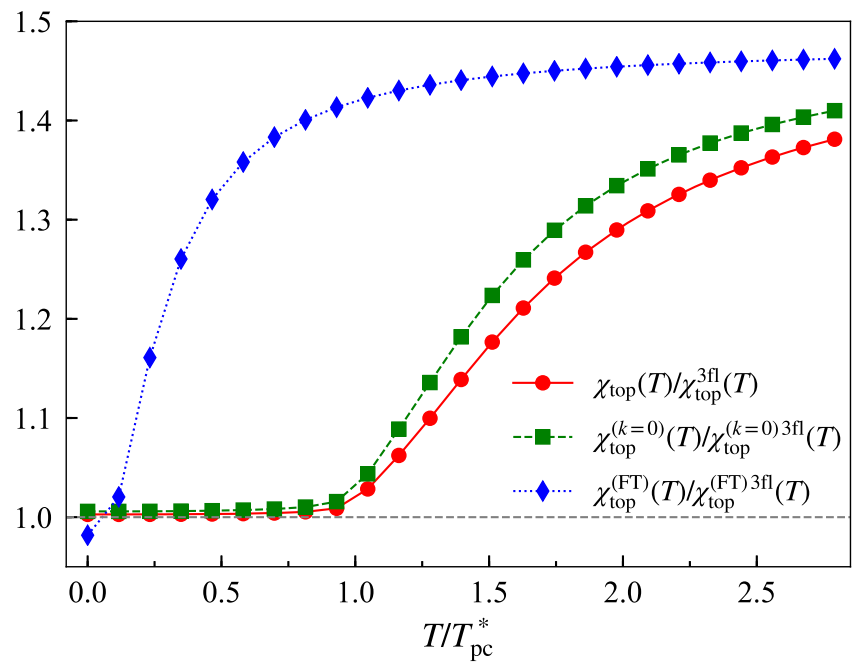

FIG. 8. The temperature dependence of the $\chi_{\text {top }}(T) / \chi_{\text {top }}^{3 \mathrm{fl}}(T)$ compared with the one without the anomaly-induced flavor breaking, the $k$-term, and the case in the free-quark theory, $\chi_{\text {top }}^{(\mathrm{FT})}(T) / \chi_{\text {top }}^{(\mathrm{FT}) 3 \mathrm{fl}}(T)$. breaking value $\left(m_{s} / m_{l}\right)^{2}$, the $\chi_{\text {top }}(T) / \chi_{\text {top }}^{3 \mathrm{fl}}(T)$ merges with the quark-FT regime, to asymptotically converge to $\chi_{\text {top }}(T) / \chi_{\text {top }}^{3 f l}(T) \simeq 3 /\left(2+m_{l} / m_{s}\right) \simeq 1.5$. Thus, the topological susceptibility gets the nonperturbative flavor breaking at around the chiral crossover criticality, which is manifestly different from the trivial-flavor breaking as seen in the quark-FT.

It is remarkable that the catalysis of the $U(1)_{A}$ breaking by the nonperturbative strange quark condensate may account for the tension in the effective restoration of the $U(1)_{A}$ symmetry currently observed on lattices with the two-flavor [13-16] and the $2+1$ flavor [17-19] near the chiral limit.

In the figure, also has been displayed comparison with the case without the anomaly-induced flavor breaking, the $k$-term. We see that the $k$-term plays a role of the destructive interference for the strange quark contribution to the $\chi_{\text {top }}$, as was seen in Fig. 4.

The panel of (a) in Fig. 9 shows the $\chi_{\text {top }}$ normalized to the vacuum value, in comparison with the ChPT prediction up to the next-to-leading order (NLO) [47] and the recent lattice data with $2+1(+1)$ flavors having a physical pion mass and the continuum limit being taken $[32,33] .{ }^{6}$ The predicted $T$ dependence (denoted by "CJT" in the figure) is actually slower-damping, and is for $T / T_{p c}^{*} \lesssim 1.5$ in good agreement with the lattice QCD data [32,33], which is not realized by the ChPT. This would manifest the importance of nonperturbative thermal contribution including the enhanced flavor breaking by $m_{s}\langle\bar{s} s\rangle(T) / m_{l}\langle\bar{l} l\rangle(T) \gg$ $m_{s} / m_{l}$ above $T_{p c}^{*}$, as depicted in Fig. 4. Substantial deviation from the lattice data can be seen for $T / T_{p c}^{*}>1.5$. The model-prediction curve would also deviate from the one derived from the dilute instanton gas approximation [48,49], which follows the lattice data [33] in whole temperature range. This can be understood as follows. The present linear-sigma model picture may break down at high $T$, where degrees of freedom of quarks (and gluons) turn to become important. One may estimate such a $T$ as $T \sim m_{q}^{\text {constituent }} \sim 330 \mathrm{MeV}$, where the latter denotes mass of constituent up and down quarks (given roughly by one-third of proton mass). This $T$ corresponds to $T / T_{p c}^{*} \sim 1.5$. Therefore, the present model prediction may be reliable only up until $T / T_{p c}^{*} \sim 1.5$. Note, however, that it is sufficient for $T / T_{p c}^{*} \lesssim 1.5$ to explore the nonperturbative flavor violation in the $\chi_{\text {top }}$, which starts to be eminent at around the chiral crossover $T / T_{p c}^{*} \gtrsim 1$.

Below the chiral crossover, $T<T_{p c}^{*}$, the present model perfectly fits with the ChPT prediction and lattice data. This implies that the chiral symmetry for light quarks is essential in the low-temperature region, as discussed in

\footnotetext{
${ }^{6}$ Note that in Ref. [32], data have been taken only for two different lattice spacings. So, continuum extrapolation in Ref. [32] may not reliably be performed to give predictions.
} 


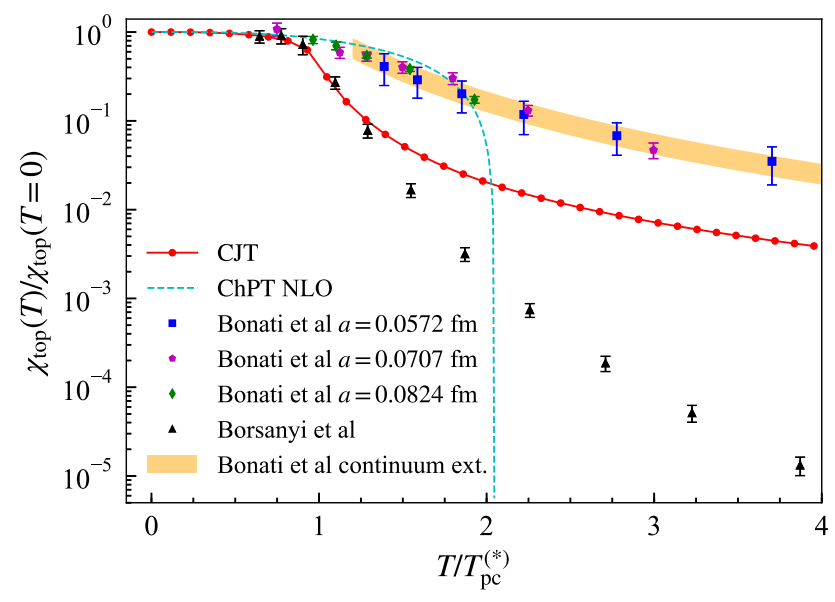

(a)

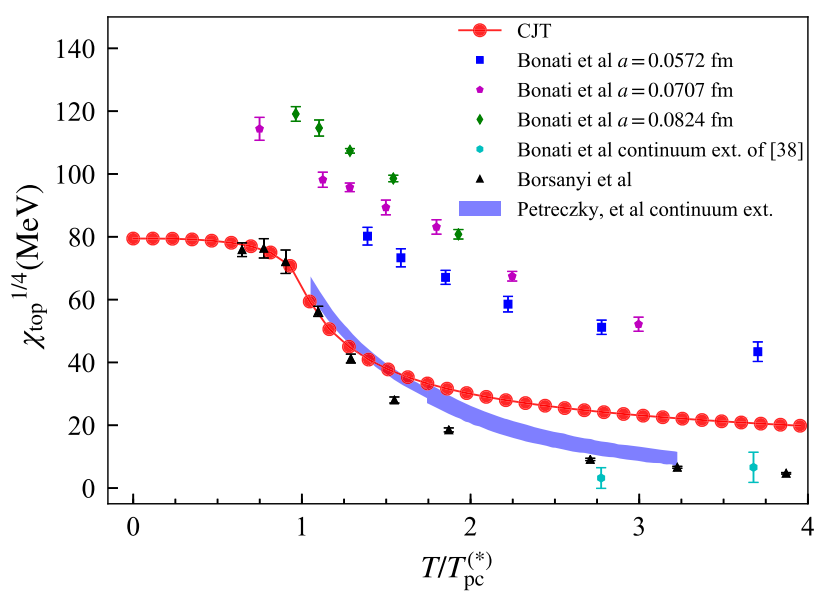

(b)

FIG. 9. (a): The comparison of the $\chi_{\text {top }}(T) / \chi_{\text {top }}(T=0)$ (labeled as "CJT" in the plot) with the ChPT prediction up to the next-toleading order (NLO) (one-loop) [47] and the lattice QCD data [32,33]. We have taken $T_{\mathrm{pc}}^{*}=215 \mathrm{MeV}$ for the CJT result, and $T_{\mathrm{pc}}=155 \mathrm{MeV}$ for the lattice simulations and the ChPT prediction. The band corresponds to the continuum extrapolation of the lattice QCD data [32], which is estimated by the function $\chi(a, T) / \chi(a, T=0)=D_{0}\left(1+D_{1} a^{2}\right)\left(T / T_{c}\right)^{D_{2}}$ with $D_{0}=1.17 D_{1}=0, D_{2}=$ -2.71 and $T_{c}=155 \mathrm{MeV}$. (b): The unnormalized topological susceptibility, $\chi_{\text {top }}{ }^{1 / 4}(\mathrm{MeV})$, versus temperature, in comparison with the continuum extrapolated results of the recent lattice simulations. The continuum extrapolated values of Bonati et al. are evaluated up to $O\left(a^{4}\right)$ corrections, where $a$ denotes the lattice scaling [38]. The band corresponds to the continuum extrapolation of the lattice QCD data [50].

the literature [47]. Note that the ChPT-governed domain is intact even if one includes the next-to-next-to-leading order (NNLO) correction in the ChPT analysis [51]. Beyond the ChPT-governed domain, above $T_{p c}^{*}$ the strange quark condensate would serve as an important source to develop the topological susceptibility, as the consequence of the nonperturbative flavor breaking.

In the panel (b) of Fig. 9, ${ }^{7}$ we also plot the temperature dependence of an unnormalized susceptibility $\chi_{\text {top }}^{1 / 4}(\mathrm{MeV})$, where comparison with some recent lattice simulations with the extrapolation to the continuum limit is available $[33,38,50]$. We see that at around the chiral crossover $T / T_{p c}^{*} \sim 1-1.5$ the CJT result is in good agreement with those continuum extrapolated data. Although qualitatively having agreement, for $T / T_{p c^{*}} \gtrsim 1.5$ the CJT result tends to predict a somewhat larger $\chi_{\text {top }}^{1 / 4}(\mathrm{MeV})$. This discrepancy gets more larger as $T / T_{p c}^{*}$ gets larger and larger. This would be subject to the model-systematic error (about 30\%) and validity of the linear sigma model description, as noted above.

To reconcile the gap observed when $T / T_{p c}^{*} \gtrsim 1.5$, one may note that model parameters in the chiral effective model can actually have intrinsic-temperature dependence,

\footnotetext{
${ }^{7}$ The result from Ref. [50] is included into the panel (b) of Fig. 9, in which a Nambu-Goldstone pion mass is $160 \mathrm{MeV}$, somewhat larger than the physical pion mass. The continuum extrapolation data on the topological susceptibility are shown to be close to the subsequent observation of another lattice simulation group [33].
}

which could mimic a part of nondecoupling effects from integrating out quarks and/or gluons. For instance, as noted in [52], the instanton study predicts the parameter $B$ in the $U(1)_{A}$ anomalous part to have an intrinsic-temperature dependence, which is relevant to the QCD topological structure at high temperatures. Thus, such an intrinsictemperature dependence might pull the CJT result down for $T>T_{p c}^{*}$, to be fully consistent with the lattice results in the continuum limit $[33,38,50]$.

\section{SUMMARY AND CONCLUSION}

In this paper, we have explored quark-flavor violation effects at high temperatures, induced from nonperturbative thermal loop corrections and axial anomaly. Working on a three-flavor linear-sigma model including an axial-anomaly induced-flavor breaking term, we employed a nonperturbative analysis following the Cornwall-Jackiw-Tomboulis formalism. It was shown that the model undergoes a chiral crossover with a pseudocritical temperature, consistently with lattice observations.

Regarding the flavor violation, what we have found is summarized as follows:

(i) Nonperturbative thermal loop corrections drive upand down-quark condensates to drop faster than the strange quark's toward the criticality, but still keep nonzero value even going far above the pseudocritical temperature. In particular, just above the criticality the flavor breaking in the quark-condensate ratio $m_{s}\langle\bar{s} s\rangle(T) / m_{l}\langle\bar{l} l\rangle(T)$ has been shown to scale much differently from a trivial flavor violation 
predicted from the ideal quark gas, and is contrast to the chiral perturbation theory predicting almost flavor-symmetric quark condensates. This manifests the nonperturbative generation of a significant flavor breaking at the chiral criticality (Fig 4);

(ii) The anomaly-related flavor-breaking effect acts as a catalyzer for the chiral restoration, while it reduces the amount of flavor breaking in the up, down and strange condensates (Fig. 2);

(iii) The meson flavor mixing structures are drastically affected in passing the chiral crossover, due to the nonperturbative thermal loop effects (Fig. 5), in which the anomaly-induced flavor breaking is found to be almost irrelevant;

(iv) The meson spectroscopy gets corrected by the net nonperturbative flavor breaking effects around and above the critical temperature (Figs. 6 and 7), where the scalar meson mass hierarchy is significantly altered by the presence of the anomaly-related flavor breaking (Eq. (4.4));

(v) Above the pseudocritical temperature, the topological susceptibility is highly enhanced due to the surviving strange quark condensate compared to the three-flavor universal limit, which cannot be detected by the chiral perturbation theory (the panel of (a) in Fig. 9). The predicted scaling in temperature significantly deviates from the dilute instanton gas prediction. There the anomaly-induced flavor breaking plays a role of the destructive interference for the enhancement, as in the flavor breaking in the quark condensates (Fig. 8).

(vi) It is noteworthy that $U(1)_{A}$ breaking perceived by the topological susceptibility is catalyzed by the nonperturbative strange quark condensate, which may account for the tension in the effective restoration of the $U(1)_{A}$ symmetry currently observed on lattices with two flavors [13-16] and $2+1$ flavors [17-19] near the chiral limit.

(vii) It is intriguing that the topological susceptibility in Eq. (2.40), reflecting the flavor-singlet nature, explicitly includes the strange quark contribution. Besides, Eq. (2.40) can be reduced to the wellknown formula $\chi_{\text {top }}=m_{l}^{2} \chi_{5 \text {,disc }}$ with keeping finite strange quark mass (See Appendix A). This implies that $\chi_{5 \text {,disc }}$ should implicitly include the strange quark contribution in the case of $2+1$ flavor QCD. Indeed, $\chi_{\text {top }}$ vanishes when either of all current quark masses goes to zero because of the flavor singlet nature, so does $\chi_{5 \text {,disc }}$ in the vanishing strange quark mass limit.

These predictions should, in a qualitative sense, be understood as new chiral critical features in hot QCD. Precise comparison with lattice simulations may be done by taking into account a possible systematic error (e.g., considering the intrinsic-temperature dependence on the model parameters), which stems from modeling lowenergy QCD as a linear sigma model description. The systematic error might be estimated by referring to the pseudocritical temperature $T_{p c}^{*}$, which we observed to be $\simeq 215 \mathrm{MeV}$, while the recent lattice simulations have reported $T_{p c} \simeq 155 \mathrm{MeV}$. The discrepancy, regarded as the systematic error of the present analysis, is about $30 \%$. (Furthermore, going beyond the Hartree approximation that we have worked on, our pseudocritical temperature might also get close to the lattice observation.) Though having such uncertainty, the present analysis would certainly grab essential features at the hot QCD criticality, e.g., the nonperturbative flavor breaking, which would be seen as a consequence of the significant deviation in the criticality scaling for the topological susceptibility from the dilute instanton gas prediction, which is on almost the same trajectory as the data from Ref. [33] in the panel (a) of Fig. 9. Comparison also with other continuum extrapolated lattice data $[33,50]$ in the panel (b) of Fig. 9 implies that around the chiral crossover $\left(T / T_{p c}^{*} \sim 1-1.5\right)$, the CJT result is in good agreement with the continuum extrapolated lattice data. Although the CJT result is deviated from the lattice results for $T / T_{\mathrm{pc}} \gtrsim 1.5$, it would sound reasonable to consider the present model to be overall on a right track the hot QCD keeps, with the possible systematic error of $30 \%$ for the present model analysis taken into account. Note also that as discussed in $[38,53]$, the reduction of various systematic uncertainties in the lattice $\chi_{\text {top }}$ measurements is still underway. In future we expect a more precise measurement of $\chi_{\text {top }}$ with the errors in the continuum band shrank even further.

The nonperturbative flavor breaking, especially, the significance of the strange quark condensate, in the topological susceptibility at around the pseudocritical temperature would give an impact on applications to QCD axion cosmological models. The epoch, in which QCD axion starts to roll and oscillate as well as the position in the potential, is crucial to estimate the relic abundance of the axion as a dark matter today. This is subject to the temperature (or time) dependence of the $\chi_{\text {top }}$ (which corresponds to the potential height) at around and/or above the chiral crossover boundary. As recently investigated in [54], below and above this crossover boundary, the twoflavor chiral perturbation theory and dilute instanton gas descriptions have separately been applied in evaluating the $\chi_{\text {top }}$ (with imposing a continuity condition between two domains through macroscopic thermodynamics quantities). Our present work would improve or refine this existing approach by including the strange quark contribution with use of the linear sigma model description, instead of the dilute instanton gas, at around and/or above the chiral crossover. This would provide a complementary evaluation of the QCD axion cosmology, with higher reliability. Detailed study is to be pursued in another publication. 
Similar significance of the nonperturbative flavor breaking can also be observed in other chiral effective models, like Nambu-Jona-Lasinio models with a nonperturbative formalism for thermal loop corrections, e.g., functional renormalization group method. This would be a crosscheck on what we have addressed in this paper, and could give some new interpretation from a different point view in terms of quark degrees of freedom and renormalization group. It would also be interesting to work along this line in the future.

Another issue to be pursued is to examine explicit dependence of the strange quark mass on the topological susceptibility in Eq. (2.40). The chiral model that we presently employed has enabled to compute only the leading order term of order of $m_{q}$, and we have confirmed the strange quark mass dependence merely in the vanishing mass limit. Next-to-leading order effects coming from the pseudoscalar susceptibility terms can be evaluated by working on other chiral models (e.g., the Nambu-Jona-Lasinio model). This would make an important step to help deeper understanding the strange quark contribution to the topological susceptibility, which could be testable on lattices.

\section{ACKNOWLEDGMENTS}

We are grateful to Massimo D'Elia, Xu-Guang Huang, and Robert Pisarski for useful comments. This work was supported in part by the National Science Foundation of China (NSFC) under Grants No. 11747308 and No. 11975108 and the Seeds Funding of Jilin University (S. M.). M. K. and A. T. thank for the hospitality of Center for Theoretical Physics and College of Physics, Jilin University where the present work has been partially done. The work of A. T. was supported by the RIKEN Special Postdoctoral Researcher program and partially by JSPS KAKENHI Grant No. JP20K14479.

\section{APPENDIX A: REDUCTION OF $\chi_{\text {top }}$ INTO $\chi_{5 \text {,dise }}$ WITH THE FLAVOR-SINGLET CONDITION}

The topological susceptibility with the flavor singlet condition [Eq. (2.40)] can be rewritten by the disconnected pseudoscalar susceptibility $\chi_{5 \text {,disc }}$ To see it, we first show the correlation between the quark condensates and the pseudoscalar susceptibilities, through the Ward identities derived in QCD [9,41].

We begin with the generic expression for the expectation value of an operator $\mathcal{O}\left(x_{1}\right)$ evaluated by the QCD generating functional,

$$
\begin{aligned}
\left\langle\mathcal{O}\left(x_{1}\right)\right\rangle= & Z_{\mathrm{QCD}}^{-1} \int\left[\Pi_{f} d q_{f} d \bar{q}_{f}\right][d A] \mathcal{O}\left(x_{1}\right) \\
& \times \exp \left(-\int_{T} d^{4} x \mathcal{L}_{\mathrm{QCD}}\right)
\end{aligned}
$$

Then we consider the chiral $S U(3)$ transformation for this expectation value. Under the $S U(3)_{A}$ flavor symmetry, the quark field transforms with the infinitesimal rotation angle $\alpha_{A}^{a}(x)$ as

$$
q(x) \rightarrow q(x)+\left(i \alpha_{A}^{a}(x) \frac{\lambda_{a}}{2} \gamma_{5} q(x)\right) \quad(\text { for } a=1, \cdots 8) .
$$

So, the $S U(3)_{A}$ transformation of $\left\langle\mathcal{O}\left(x_{1}\right)\right\rangle$ in Eq. (A1) goes like

$$
\begin{gathered}
\int_{T} d^{4} x\left\langle\frac{\partial \mathcal{O}^{\prime}\left(x_{1}\right)}{\partial \alpha_{A}^{a}(x)}\right\rangle+\int_{T} d^{4} x\left\langle i \mathcal{O}\left(x_{1}\right) \partial_{\mu}^{(x)} j_{5}^{a \mu}(x)\right\rangle \\
+\int_{T} d^{4} x\left\langle\mathcal{O}\left(x_{1}\right)\left(\bar{q}\left\{\frac{\lambda_{a}}{2}, \mathcal{M}\right\} \gamma_{5} q(x)\right)\right\rangle=0,
\end{gathered}
$$

where integration over space-time $(T)$ has been taken, and $\mathcal{O}^{\prime}\left(x_{1}\right)$ is the transformed operator and $j_{5}^{a \mu}$ represents the axial current, $j_{5}^{a \mu}(x)=\bar{q} \gamma^{\mu} \gamma_{5} \frac{\lambda_{a}}{2} q(x)$. Equation (A3) is the Ward identity for $S U(3)$ chiral symmetry.

The second term in the left-hand side of Eq. (A3) is evaluated as

$$
\begin{aligned}
\int_{T} d^{4} x\left\langle i \mathcal{O}\left(x_{1}\right) \partial_{\mu}^{(x)} j_{5}^{a \mu}(x)\right\rangle & =\int_{T} d^{4} x \partial_{\mu}^{(x)}\left\langle 0\left|T i \mathcal{O}\left(x_{1}\right) j_{5}^{a \mu}(x)\right| 0\right\rangle \\
& = \begin{cases}\left\langle 0\left|\left[i Q_{5}^{a}\left(x_{1}^{0}\right), \mathcal{O}\left(x_{1}\right)\right]\right| 0\right\rangle=-\int_{T} d^{4} x\left\langle\frac{\partial \mathcal{O}^{\prime}\left(x_{1}\right)}{\partial \alpha_{A}^{a}(x)}\right\rangle & \text { (Chiral limit) } \\
0 & \text { (Explicit chiral symmetry breaking), }\end{cases}
\end{aligned}
$$

where $Q_{5}^{a}$ is the $S U(3)_{A}$ charge defined as $Q_{5}^{a}\left(x^{0}\right) \equiv$ $\int d^{3} x j_{5}^{a \mu=0}(x)$. In the chiral limit this term does not drop even though it is just a surface term, due to emergence of the massless NG (Nambu-Goldstone) bosons associated with the spontaneous chiral symmetry breaking, which survive in the infinitely longdistance $\left(\left|x_{1}-x\right| \rightarrow \infty\right)$. In contrast, the explicit chiral symmetry breaking gives NG bosons nonzero 
masses, so that $\left\langle 0\left|\operatorname{TiO}\left(x_{1}\right) j_{5}^{a \mu}(x)\right| 0\right\rangle$ goes to zero when $\left|x_{1}-x\right| \rightarrow \infty$.

Now we choose the $\mathcal{O}\left(x_{1}\right)$ as the pseudoscalar operator,

$$
\mathcal{O}^{b}\left(x_{1}\right)=i q \gamma_{5} \frac{\lambda^{b}}{2} q\left(x_{1}\right), \quad(\text { for } b=0,1, \ldots, 8) .
$$

Then, for the $\pi$-channel corresponding to $\mathcal{O}^{b=1,2,3}\left(x_{1}\right)$ with $\alpha_{A}^{a=1,2,3}(x)$, the Ward identity in Eq. (A3) for this operator reads

$$
\left\langle\bar{q}_{l} q_{l}\left(x_{1}\right)\right\rangle=-m_{l} \chi_{\pi}\left(x_{1}\right),
$$

where $q_{l}$ is the light quark field, $q_{l}=(u, d, 0)$, and $\chi_{\pi}$, called the pseudoscalar susceptibility, is defined as

$$
\chi_{\pi}\left(x_{1}\right)=\int_{T} d^{4} x\left\langle\left(i \bar{q}_{l} \gamma_{5} q_{l}\left(x_{1}\right)\right)\left(i \bar{q}_{l} \gamma_{5} q_{l}(x)\right)\right\rangle_{\mathrm{con}},
$$

with $\langle\cdots\rangle_{\text {con }}$ representing the connected part of the correlation function. Thus, one finds that the light quark condensate is connected with the pseudoscalar susceptibility $\chi_{\pi}[9]$.

As for the $\eta-\eta^{\prime}$ channel, corresponding to $\mathcal{O}^{b=0}\left(x_{1}\right)$ with $\alpha_{A}^{a=8}(x)$ and $\mathcal{O}^{b=8}\left(x_{1}\right)$ with $\alpha_{A}^{a=8}(x)$, one can also find the correlations between the quark condensates and the pseudoscalar susceptibilities from the associated Ward identities [9],

$$
\begin{aligned}
& \left\langle\bar{q}_{l} q_{l}\right\rangle-2\langle\bar{s} s\rangle=-m_{l}\left(\chi_{P}^{l l}+\chi_{P}^{l s}\right)+2 m_{s}\left(\chi_{P}^{s s}+\chi_{P}^{l s}\right), \\
& \left\langle\bar{q}_{l} q_{l}\right\rangle+4\langle\bar{s} s\rangle=-m_{l}\left(\chi_{P}^{l l}-2 \chi_{P}^{l s}\right)-2 m_{s}\left(2 \chi_{P}^{s s}-\chi_{P}^{l s}\right),
\end{aligned}
$$

where $\chi_{P}^{l l}, \chi_{P}^{s s}$, and $\chi_{P}^{l s}$ respectively are defined as

$$
\begin{aligned}
\chi_{P}^{l l} & =\int_{T} d^{4} x\left\langle\left(i \bar{q}_{l} \gamma_{5} q_{l}\left(x_{1}\right)\right)\left(i \bar{q}_{l} \gamma_{5} q_{l}(x)\right)\right\rangle, \\
\chi_{P}^{s s} & =\int_{T} d^{4} x\left\langle\left(i \bar{s} \gamma_{5} s\left(x_{1}\right)\right)\left(i \bar{s} \gamma_{5} s(x)\right)\right\rangle, \\
\chi_{P}^{l s} & =\int_{T} d^{4} x\left\langle\left(i \bar{q}_{l} \gamma_{5} q_{l}\left(x_{1}\right)\right)\left(i \bar{s} \gamma_{5} s(x)\right)\right\rangle .
\end{aligned}
$$

From the correlation relations in Eq. (A8), the light quark condensate and the strange quark condensate can be read as

$$
\begin{aligned}
\left\langle\bar{q}_{l} q_{l}\right\rangle & =-m_{l} \chi_{P}^{l l}+2 m_{s} \chi_{P}^{l s}, \\
\langle\bar{s} s\rangle & =-m_{s} \chi_{P}^{s s}+\frac{m_{l}}{2} \chi_{P}^{l s} .
\end{aligned}
$$

In addition, by using Eqs. (A6) and (A8), $\chi_{P}^{l s}$ can be written as the disconnected pseudoscalar susceptibility [41]:

$$
\chi_{P}^{l s}=\frac{m_{l}}{2 m_{s}}\left(\chi_{P}^{l l}-\chi_{\pi}\right)=2 \frac{m_{l}}{m_{s}} \chi_{5, \mathrm{disc}} .
$$

Using Eqs. (A10) and (A11), one can now find that the topological susceptibility in Eq. (2.40) is reduced to the disconnected pseudoscalar susceptibility:

$$
\begin{aligned}
\chi_{\text {top }} & =\left(\frac{\left\langle\bar{q}_{l} q_{l}\right\rangle(T)}{m_{l}}+\frac{\langle\bar{s} s\rangle(T)}{m_{s}}\right) \bar{m}^{2}+\bar{m}^{2}\left(\chi_{P}^{l l}+2 \chi_{P}^{l s}+\chi_{P}^{s s}\right) \\
& =\frac{1}{2} m_{l} m_{s} \chi_{P}^{l s} \\
& =m_{l}^{2} \chi_{5, \text { disc }} .
\end{aligned}
$$

Thus the topological susceptibility in Eq. (2.40) exactly reproduces the well-known formula described by $\chi_{5 \text {,disc }}$ in the $2+1$-flavor QCD. Note that in this derivation we have not assumed the strange quark to be heavy enough $\left(m_{s} \rightarrow \infty\right)$, nor make the assumption of a nearly-exact chiral-symmetry for up and down quarks where the chiral condensate is estimated to be negligible.

\section{APPENDIX B: QUARK CONDENSATE IN FREE THEORY}

In the free theory of quark fields at finite temperature, the quark condensate is given as

$$
\langle\bar{q} q\rangle_{\mathrm{FT}}=-i 4 m_{q} \int \frac{d^{4} p}{(2 \pi)^{4}} \frac{1}{p^{2}-m^{2}+i \epsilon}+\frac{4 m_{q} T^{2}}{2 \pi^{2}} I\left(m_{q}\right)
$$

where

$$
I\left(m_{q}\right)=\int_{0}^{\infty} d x \frac{x^{2}}{\sqrt{x^{2}+m_{q}^{2} / T^{2}}} \frac{1}{1+\exp \left(\sqrt{x^{2}+m_{q}^{2} / T^{2}}\right)} .
$$

For $T \gg m_{q}$, the second term of Eq. (B1) goes like

$$
\frac{4 m_{q} T^{2}}{2 \pi^{2}} \int_{0}^{\infty} d x \frac{x}{1+e^{x}} .
$$

Focusing only on the thermal quark loop, the ratio of the strange quark condensate to the light quark's is evaluated as

$$
\frac{\langle\bar{s} s\rangle_{\mathrm{FT}}(T)}{\langle\bar{l} l\rangle_{\mathrm{FT}}(T)}=\frac{m_{s}}{m_{l}} \frac{I\left(m_{s}\right)}{I\left(m_{l}\right)},
$$

Since $I\left(m_{l}\right)=I\left(m_{s}\right)$ for $T \gg m_{q}$, the ratio of quark condensates converges to $m_{s} / m_{l}$ as the temperature increases. 
[1] Y. Aoki, G. Endrodi, Z. Fodor, S. D. Katz, and K. K. Szabo, Nature (London) 443, 675 (2006).

[2] S. Borsanyi, G. Endrodi, Z. Fodor, C. Hoelbling, S. Katz, S. Krieg, C. Ratti, and K. K. Szabo (Wuppertal-Budapest Collaboration), J. Phys. Conf. Ser. 316, 012020 (2011).

[3] H.-T. Ding, F. Karsch, and S. Mukherjee, Int. J. Mod. Phys. E 24, 1530007 (2015).

[4] A. Bazavov et al. (HotQCD Collaboration), Phys. Lett. B 795, 15 (2019).

[5] H.-T. Ding, Nucl. Phys. A1005, 121940 (2021).

[6] R. D. Pisarski and F. Wilczek, Phys. Rev. D 29, 338 (1984).

[7] C. McNeile, A. Bazavov, C. T. H. Davies, R. J. Dowdall, K. Hornbostel, G. P. Lepage, and H. D. Trottier, Phys. Rev. D 87, 034503 (2013).

[8] M. Cheng et al., Phys. Rev. D 77, 014511 (2008).

[9] A. Gómez Nicola and J. Ruiz de Elvira, J. High Energy Phys. 03 (2016) 186.

[10] M. Kawaguchi, S. Matsuzaki, and A. Tomiya, Phys. Lett. B 813, 136044 (2021).

[11] Y. Kuroda, M. Harada, S. Matsuzaki, and D. Jido, Prog. Theor. Exp. Phys. 2020, 053D02 (2020).

[12] J. M. Cornwall, R. Jackiw, and E. Tomboulis, Phys. Rev. D 10, 2428 (1974).

[13] G. Cossu, S. Aoki, H. Fukaya, S. Hashimoto, T. Kaneko, H. Matsufuru, and J.-I. Noaki, Phys. Rev. D 87, 114514 (2013); 88, 019901(E) (2013).

[14] A. Tomiya, G. Cossu, S. Aoki, H. Fukaya, S. Hashimoto, T. Kaneko, and J. Noaki, Phys. Rev. D 96, 034509 (2017); 96, 079902(A) (2017).

[15] K. Suzuki, S. Aoki, Y. Aoki, G. Cossu, H. Fukaya, and S. Hashimoto (JLQCD Collaboration), Proc. Sci., CD2018 (2019) 085 [arXiv:1908.11684].

[16] K. Suzuki, S. Aoki, Y. Aoki, G. Cossu, H. Fukaya, S. Hashimoto, and C. Rohrhofer (JLQCD Collaboration), Proc. Sci., LATTICE2019 (2020) 178 [arXiv:2001.07962].

[17] A. Bazavov et al. (HotQCD Collaboration), Phys. Rev. D 86, 094503 (2012).

[18] M. I. Buchoff et al., Phys. Rev. D 89, 054514 (2014).

[19] H. Ding et al., Phys. Rev. Lett. 123, 062002 (2019).

[20] M. Kobayashi and T. Maskawa, Prog. Theor. Phys. 44, 1422 (1970).

[21] M. Kobayashi, H. Kondo, and T. Maskawa, Prog. Theor. Phys. 45, 1955 (1971).

[22] G. 't Hooft, Phys. Rev. Lett. 37, 8 (1976).

[23] G. 't Hooft, Phys. Rev. D 14, 3432 (1976); 18, 2199(E) (1978).

[24] V. Baluni, Phys. Rev. D 19, 2227 (1979).

[25] Y.-Y. Mao and T.-W. Chiu (TWQCD Collaboration), Phys. Rev. D 80, 034502 (2009).

[26] J. E. Kim and G. Carosi, Rev. Mod. Phys. 82, 557 (2010); 91, 049902(E) (2019).
[27] H. Leutwyler and A. V. Smilga, Phys. Rev. D 46, 5607 (1992).

[28] J. T. Lenaghan, D. H. Rischke, and J. Schaffner-Bielich, Phys. Rev. D 62, 085008 (2000).

[29] D. Roder, J. Ruppert, and D. H. Rischke, Phys. Rev. D 68, 016003 (2003).

[30] J. T. Lenaghan and D. H. Rischke, J. Phys. G 26, 431 (2000).

[31] M. Tanabashi et al. (Particle Data Group), Phys. Rev. D 98, 030001 (2018).

[32] C. Bonati, M. D’Elia, M. Mariti, G. Martinelli, M. Mesiti, F. Negro, F. Sanfilippo, and G. Villadoro, J. High Energy Phys. 03 (2016) 155.

[33] S. Borsanyi et al., Nature (London) 539, 69 (2016).

[34] E. Vicari and H. Panagopoulos, Phys. Rep. 470, 93 (2009).

[35] M. Lcscher, J. High Energy Phys. 08 (2010) 071; 03 (2014) 092(E).

[36] C. Alexandrou, A. Athenodorou, and K. Jansen, Phys. Rev. D 92, 125014 (2015).

[37] C. Alexandrou, A. Athenodorou, K. Cichy, A. Dromard, E. Garcia-Ramos, K. Jansen, U. Wenger, and F. Zimmermann, Eur. Phys. J. C 80, 424 (2020).

[38] C. Bonati, M. D’Elia, G. Martinelli, F. Negro, F. Sanfilippo, and A. Todaro, J. High Energy Phys. 11 (2018) 170.

[39] Y. Aoki, S. Borsanyi, S. Durr, Z. Fodor, S. D. Katz, S. Krieg, and K. K. Szabo, J. High Energy Phys. 06 (2009) 088.

[40] A. Bazavov et al., Phys. Rev. D 100, 094510 (2019).

[41] A. Gómez Nicola and J. Ruiz de Elvira, Phys. Rev. D 97, 074016 (2018).

[42] T. Bhattacharya et al., Phys. Rev. Lett. 113, 082001 (2014).

[43] K. Fukushima, K. Ohnishi, and K. Ohta, Phys. Rev. C 63, 045203 (2001).

[44] Y. Jiang and P. Zhuang, Phys. Rev. D 86, 105016 (2012).

[45] Y. Jiang, T. Xia, and P. Zhuang, Phys. Rev. D 93, 074006 (2016).

[46] Z.-Y. Lu and M. Ruggieri, Phys. Rev. D 100, 014013 (2019).

[47] G. Grilli di Cortona, E. Hardy, J. Pardo Vega, and G. Villadoro, J. High Energy Phys. 01 (2016) 034.

[48] D. J. Gross, R. D. Pisarski, and L. G. Yaffe, Rev. Mod. Phys. 53, 43 (1981).

[49] T. Schafer and E. V. Shuryak, Rev. Mod. Phys. 70, 323 (1998).

[50] P. Petreczky, H. P. Schadler, and S. Sharma, Phys. Lett. B 762, 498 (2016).

[51] A. Gómez Nicola, J. Ruiz De Elvira, and A. VioqueRodríguez, J. High Energy Phys. 11 (2019) 086.

[52] R. D. Pisarski and F. Rennecke, Phys. Rev. D 101, 114019 (2020).

[53] H.-T. Ding, Proc. Sci., LATTICE2016 (2017) 022 [arXiv: 1702.00151].

[54] J. E. Kim and S.-J. Kim, Phys. Lett. B 783, 357 (2018). 